\title{
The Value of Network Information
}

\author{
Itay P. Fainmesser and Andrea Galeotti*
}

December 2, 2013

\begin{abstract}
The business model of companies such as Facebook, MySpace, and Twitter, relies on monetizing the information on the interactions and influences of their users. How valuable is such information, and is its use beneficial or detrimental for consumer welfare? We study these questions in a model where a monopoly sells a network good and may price discriminate using network information: information on consumers influences and/or on consumers susceptibilities to influence. Our framework incorporates a rich set of market products, including goods characterized by global and local network effects. We derive results on the value of network information and determine under which conditions, relative to uniform price, consumer surplus increases. We demonstrate the applicability of our framework using survey data on various types of relationships.
\end{abstract}

*Fainmesser: Department of Economics, Brown University, Providence, RI 02912 (email: Itay_Fainmesser@Brown.edu); Galeotti: Department of Economics, University of Essex, Wivenhoe Park, Colchester CO4 3S, United Kingdom (email: agaleo@essex.ac.uk). We thank Matt Jackson, Shachar Kariv, Jacob Leshno, Markus Mobius, and participants to seminars at The University of California and Berkeley, Stanford University, Brown University, Microsoft Research New England, Tel Aviv University, Ohio State University, and The University of Essex, for valuable comments. We thank Neil Thakral, Michael Thaler, and Stephen Nei for excellent research assistance. Itay Fainmesser is greatful to the Economics Department at Stanford University for their hospitality and financial support during the academic year 2013-14. Andrea Galeotti is grateful to the European Research Council for support through ERC-starting grant (award no. 283454) and to The Leverhulme Trust for support through the Philip Leverhulme Prize. 


\section{Introduction}

We study the value of information about consumers' influence of each other for a monopoly selling a network good. Consider a product that has a collaborative component, such as membership in social club, the telephone, video-conference technology, an online game, a social network website, a file sharing tool, or a creative collaborative software. A potential consumer decides whether and how much to consume of the product based on her expectations of the consumptions of other consumers with whom she expects to interact: friends, colleagues, collaborators, or competitors. Now consider a monopoly selling the product. Having precise information about patterns of network externalities allows the monopoly to set different prices for different consumers. This paper characterizes how much a monopoly can increase its profit by exploiting individual level data on "who influences whom" when making its pricing decision, and the corresponding impact on consumer welfare.

Depending on the product of interest, network externalities can take different forms. When choosing her consumption, an individual may be affected by the consumption of the entire population-global network externalities-or by the consumption of a subset of the populationlocal network externalities. Moreover, recent empirical studies reveal that consumers may be heterogenous in terms of influence. There are consumers who are very influential, and there are consumers who are very susceptible to influence. In general, these may or may not be the same consumers. ${ }^{1}$

Our model of social influence incorporates this rich set of environments in a tractable framework. We model influence of a consumer by her in-degree and out-degree. The out-degree parametrizes the susceptibility to influence of the consumer. The in-degree parametrizes the level of influence of the consumer. When network externalities are local, the out-degree is the number of other consumers whom a consumer is influenced by, and the in-degree is the number of other consumers whom the consumer influences. The pattern of network effects in the market is then parsimoniously summarized by the in- and out-degree distributions of an underlying network of interactions. In the case of global externalities, each consumer cares about the weighted average consumption of the entire population, but with different intensities, as specified by her out-degree and everyone else's in-degrees.

We consider a two-stage game. In the first stage the monopoly chooses a price scheme. In the second stage, consumers observe the price scheme and make simultaneous consumption decisions, taking into account the resulting network externalities. The ability to price discriminate depends on the monopoly knowledge about the in-degree and out-degree of each consumer. The benchmark case is one where the monopolist only knows the in-degree and

\footnotetext{
${ }^{1}$ Tucker (2008), in a study of the adoption of video-conferencing technology in a large multi-national organization, shows that adoption and usage of the technology by employees is influenced by the usage of the technology by their superiors, but less so by their subordinates. Nair et al. (2010) report asymmetric influence between prominent physicians and non-specialist physicians in the context of prescription and adoption of new drugs. Aral and Walker (2012), in a study of adoption of an application in Facebook.com, also shows the importance to distinguish between influential and susceptible consumers. Goldenberg et al. (2009), in a study of adoption of pictures and video-clips in a social networking website, show that influence and susceptibility to influence are both important dimensions to understand diffusion and to identify hubs.
} 
out-degree distributions. We then consider the case in which the monopolist has more detailed information: she knows the in-degree of each consumer, the out-degree of each consumer, and, finally, both. We compare these different scenario and assess how the information that the monopoly has about the pattern of network externalities shape the optimal pricing strategy, the profits the monopoly can hope to earn, and consumers' welfare.

Our first insight is that to evaluate the value of information on in-degrees and out-degrees, it is sufficient to have information on the average level of influence and the dispersion of in-degrees and out-degrees. ${ }^{2}$ This gives a precise guide to practitioners in terms of prioritizing their data collection/purchase decisions. It also allows a company, like Facebook.com, MySpace, Google and alike, which owns data on social interactions and influence, to provide firms with sufficient statistics that convey the value of the data without revealing the data itself - also known as zero-knowledge information transmission in the Cryptology literature. ${ }^{3}$ Moreover, our result could be used to improve the pricing of stocks in IPOs of companies such as Facebook, Twitter, Lore.com or Pinterest, whose business models rely, at least in part, in monetizing the information of their users, including their interactions and influences. ${ }^{4}$

There is now convincing evidence that influence among consumers differs across markets. In some product markets the level of influence is much higher than in others, and such differences are, in part, explained by the demographic characteristics of the representative consumer of that particular market, see, e.g., Leskovec, Adamic, and Huberman (2007), Keller, Fay, and Berry (2007), Bearden and Etzel (1982). ${ }^{5}$ Our second insight then relates the value of information to general statistics of social influence. We show that the value of having information on in-degrees and/or out-degrees increases with the level of social influence and with the dispersion of the in- and out-degree distributions. We show that information on in-degrees is more valuable than information on out-degrees if, and only if, the in-degree distribution is more dispersed than the out-degree distribution. This formalizes a recurrent and important theme in marketing stressing the importance of strategies that take advantage of structural differentiation, see Krackhardt (1996).

Our analysis raises a natural question: How would consumers' welfare change if firms incorporate information on consumers' influence in their selling strategies? Indeed, there is a

\footnotetext{
${ }^{2}$ Precisely, the value of information on, say, in-degrees, is the increase in monopoly profits obtained by price discriminating on in-degrees relative to uniform pricing.

${ }^{3}$ For a classic example, see Feige et al. (1988)

${ }^{4}$ The development of new methods to identify influential and susceptible consumers from large data sets is a very active area of research in the intersection between the area of business and of information system engineering. We refer to Probst et al (2013) for a recent survey of this literature. Companies like Google are also increasing their effort in developing algorithms to identify influential consumers, see Green (2008).

${ }^{5}$ Bearden and Etzel (1982) shows that the level of influence among consumers increases with the "conspicuousness" of the product. The level of influence among consumers is also higher for products that are "consumed publicly". Leskovec, Adamic, and Huberman (2007) shows that the level of social interactions-how much buyers recommend their products- and the degree of influence- how often recommendations lead to adoption- changes greatly across product categories: high recommendation and moderate influence for DVD, moderate recommendation but high influence for books and Music, and very low recommendation and influence for Video. Keller, Fay, and Berry (2007) report that both the average level and dispersion of influence, measured by tracking word of mouth communication, is correlated to some demographic: it is much higher among teenagers as compared to the average in the population at large.
} 
current debate about whether antitrust authorities should re-focus their present investigations about Google on how Google's control of large data set about consumers' behaviour may entrench monopoly power and harm consumers' welfare, see, e.g., Newman (2013). This issue is part of a more general debate about to what extent law should facilitate informational privacy given the technological development leading private and state organisations to enjoy unprecedented abilities to collect personal data, see, M. Froomkin (2000). We show that discrimination on in-degree and on out-degree have different implications for both aggregate consumer surplus and payoff distribution across consumers. In particular, moving from nodiscrimination to price discrimination on out-degrees increases the welfare of all consumers, only if the out-degree distribution is sufficiently dispersed. Otherwise, it increases the welfare of consumers with low out-degrees and decreases the welfare of consumers with high out-degrees and the overall effect on aggregate consumer surplus is ambiguous. On the other hand, a move from no-discrimination to price discrimination on in-degrees increases unambiguously overall consumer surplus, but some consumers may be worse off.

Building on the generality of our framework we study how the monopoly's profit and the value of information are affected by the correlation in the population between consumers' indegrees and their out-degrees - henceforth degree correlation. Negative correlation between in-degree and out-degree is typical of many goods, such as [1] goods that have an element of expertise, like prescription choices by physicians, in which case few experts act as opinion leaders; [2] professional goods adopted in superior-inferior relationships, like communication devices in hierarchical organizations; and [3] fashionable goods, like design clothes, where brands are often endorsed by celebrities. ${ }^{6}$ Positive correlation between influence and susceptibility is, instead, typical of social goods in which social interaction is more symmetric, like Facebook.com usage time, and communication products, like communication applications on smartphone.

We show that an increase in degree correlation increases the monopoly profit for any pricing regime, but decreases the value of information on consumers' in- and out-degrees. That is, if influential individuals are also highly susceptible to influence, the monopoly can expect high profits, and has less scope for price discrimination. Moreover, when degree correlation is positive or moderately negative, the value of information of in-degrees and out-degrees exhibit complementarity - that is, the value of information on in-degrees is higher to a monopolist who already holds the information on out-degrees, and vice versa. Therefore, if collecting information on in-degrees and on out-degrees require identical investments, a monopoly will either gather the complete individual level data, or gather no data at all. In contrast, if degree correlation is sufficiently negative - i.e., influential individuals are less susceptible to influence, the value of information on in-degrees and out-degrees no longer exhibits complementarity and instead exhibits substitutability.

With the theoretical underpinning in hand, we proceed to illustrate novel aspects of our analysis. We consider data from a survey conducted in 75 villages in rural India (see Banerjee

\footnotetext{
${ }^{6}$ We refer to Tucker (2008), Nair et al (2010) and Aral and Walker (2012), discussed previously, for examples in which influence and susceptibility to influence are negative correlated.
} 
et al. 2011). The data documents a variety of relationships between individuals within each village. We focus on a number of different networks induced by the different relationships, which are presumably related to consumption externality of different types of goods. Thus, by exploiting the differences between the networks, we illustrate how the monopoly profit, the value of information, the consumers' welfare, and the level of complementarity of information on in- and out-degrees vary across the networks and product types.

Our paper relates to the classical literature on network externalities and network industries initiated in the 1980's by Farrell and Saloner (1985) and Katz and Shapiro (1985), and most recently extended to settings with local network externalities, e.g., Sundarajan (2006), Ballester et al. (2006), Galeotti et al. (2010) and Bramoulle et al. (2013). Our second stage can be interpreted both as a game with local externalities and as a game with global externalities. For the former interpretation, we make the realistic assumption that when taking consumption decisions consumers know their own in-degree and out-degree, but have incomplete information about the level of interactions/influence of other consumers, similarly to Galeotti et al. (2010). For the latter interpretation, the underlying assumption is that consumers care about a weighted average consumption and the weights are given by in-degrees and out-degrees.

Our main interest is, however, understanding how the monopolist can exploit the presence of such network externalities. The literature of optimal marketing strategies in the presence of social influence is quite recent and a very active field of research in economics, marketing, and computer science. Most of the literature has focused on optimal advertising and seeding strategies (distributing, initially, products for free to key consumers) given an exogenous process of product diffusion, e.g., Galeotti and Goyal (2009), Campbell (2013), Goyal and Kearns (2012), Dominigos and Richardson (2001), and Kempe et al. (2003).

Only few papers study optimal pricing as marketing strategies in environments with network influence. Hartline et al. (2008) and Arthur et al. (2009) consider dynamic optimal pricing in a setting where adoption is sequential and consumers are myopic. They show that the optimal dynamic pricing strategy is NP-hard to compute, and develop a simple pricing strategy to illustrate the potential value of exploiting influences across consumers. Candogan et al. (2012) and Bloch and Querou (2013) are the closest studies to our. They consider monopoly pricing when both the monopolist and the consumers have perfect knowledge of the architecture of the network of influence. Their aim is to determine when the optimal pricing strategy reflects the centrality measures of the consumers in the network. While we share with this work the basic motivation, our research questions and method of analysis are very distinctive. In particular, our framework allows to evaluate the value of information on consumers' influence for the monopoly, and the effect that the use of such information may have on consumers' welfare. The emphasis that our work places on these questions is, to the best of our knowledge, novel in the literature. 


\section{Model}

There is a single monopoly, which can produce any quantity of a divisible good at no cost, and a unit measure of potential consumers $N=[0,1]$. Consumers' behavior is influenced by the consumptions of other consumers (network externalities) and these influences can be heterogeneous across consumers. In the first stage of the game the monopoly chooses a price scheme $\mathbf{p}=\left\{p_{i}\right\}_{i \in N}$. The ability of the monopoly to assign different prices to different consumers depend on her knowledge about the network. In the second stage, consumers observe the price scheme and make simultaneous consumption decisions, $\mathbf{x}=\left\{x_{i}\right\}_{i \in N}$, taking into account the resulting network externalities. We emphasize that the model can be modified with minor changes to capture a discrete good which each consumer can choose either to adopt or not to adopt - our analysis and results follow through with no change. See section 2.1.1 for more details.

We now formalize the ingredients of our model: patterns of influence, network externalities, and different level of network knowledge of the monopoly. The levels of influence of a consumer are parametrized by her out-degree, $k$, and in-degree, $l$. A consumer with out-degree $k$ draws, after making her consumption decision, $k$ others and each consumer with in-degree $l$ is drawn by $l$ others. ${ }^{7}$ A fraction $P(k)$ of consumers has out-degree $k$, and a fraction $H(l)$ of consumers has in-degree $l$, where $l, k \in D=\left\{0, \ldots, k^{\max }\right\}$, and $\sum_{k \in D} P(k)=\sum_{l \in D} H(l)=1$. We assume for now that there is no correlation in the population between a consumer's in-degree and her out-degree and vice-versa, that is, $P(k \mid l)=P(k)$ and $H(l \mid k)=H(l)$; section 5 studies the case with correlation. Consistency requires that the average out-degree equals the average in-degree, i.e., $\sum P(k) k=\hat{k}=\sum H(l) l$. We refer to $\hat{k}$ as to the average level of influence. We assume that the different draws of a consumer, as well as the draws of different consumers are independent of each other. Therefore, if $i$ samples $j$, the probability that $j$ is sampled by $l-1$ others is $\bar{H}(l)=\frac{1}{\widehat{k}} H(l) l$.

Consumers have quadratic utility that exhibits network externalities. ${ }^{8}$ For a given profile of consumption $\mathbf{x}=\left(x_{i}, \mathbf{x}_{-i}\right)$ and unit price $p_{i}$, the utility of consumer $i$ who benefits from interacting with a finite set of consumers $N_{i}$ (i.e., draws the set of consumers $N_{i}$ ) is

$$
u_{i}\left(x_{i}, \mathbf{x}_{-i}, p_{i}\right)=x_{i}-\frac{1}{2} x_{i}^{2}+\gamma \sum_{j \in N_{i}} x_{i} x_{j}-p_{i} x_{i}
$$

where $\gamma>0$ is the positive network externalities coefficient. Since different draws of a con-

\footnotetext{
${ }^{7}$ More precisely, a consumer with out-degree $k$ draws $k$ other consumers according to an atomless weighted-uniform distribution on the unit interval, where the weights are determined by the in-degrees of the sampled consumers. Therefore, the unconditional probability distribution function assigns to a consumer with in-degree $l$ a density for being sampled that is $l$ times higher than it assigns for a consumer with in-degree 1.

${ }^{8}$ Beyond their mathematical appeal, the classic motivation for the assumption of quadratic utility functions is that it provides a second order taylor approximation for other utility functions and thus captures first (and second) order effects and neglect only higher order effects of a smaller magnitude. Furthermore, our analysis and results carry over to a more general class of utility functions that induce linear best responses by consumers (see Bramoullé et al. 2011 for discussion). The only exception to this last point is that in order to make any claim on aggregate consumer surplus, we cannot remain agnostic with respect to the utility function that we choose.
} 
sumer, as well as the draws of different consumers, are independent of each other, we have that for given $\left(x_{i}, \mathbf{x}_{-i}\right)$ the expected utility to consumer $i$ is

$$
U_{i}\left(x_{i}, \mathbf{x}_{-i}, p_{i}, k\right)=\left(1-p_{i}\right) x_{i}-\frac{1}{2} x_{i}^{2}+\gamma x_{i} k A\left(\mathbf{x}_{-i}\right)
$$

where $A\left(\mathbf{x}_{-i}\right) \triangleq E\left[x_{j} \mid j \in N_{i}\right]$ is the average consumption across samples of consumers. Given $A\left(\mathbf{x}_{-i}\right)$, the consumption of consumer $i$ depends on her type only via her out-degree $k$ and the price $p_{i}$. Therefore, for any pricing scheme for which the price $p_{i}$ depends at most on the outdegree and in-degree of consumer $i$, we can write the demand of a consumer with out-degree $k$ and in-degree $l$ as $x(k, l)$; and

$$
A\left(\mathbf{x}_{-i}\right)=\sum_{l} \bar{H}(l) \sum_{k} P(k) x(k, l) .
$$

Before solving the monopoly's problem, we study the consumption equilibrium induced by the consumers' decisions in the second stage of the game given any pricing scheme chosen by the monopoly. The following result establishes conditions under which, for any pricing scheme chosen by the monopoly, there exists a unique consumption equilibrium. The result also provides a characterization of equilibrium consumptions levels.

Proposition 1. Let $\mathbf{p}$ be any price schedule set by the monopoly in the first stage of the game and specifying for each consumer $i$ a price per unit $p_{i} \in \mathbb{R}$.

1. If $\gamma k^{\max }<1$, then, in the consumption stage, there exists a unique Bayes-Nash equilibrium $\mathbf{x}^{*}$.

2. Assume that according to $\mathbf{p}$ any two consumers with identical in-and out-degrees are priced the same. Then, in the unique equilibrium of the consumption stage any two consumers with identical in- and out-degrees make the same consumption decision. In particular, let $p(k, l)$ and $x(k, l)$ be the price and the demand of a consumer with outdegree $k$ and in-degree $l$. Then, in the unique equilibrium of the consumption stage

$$
x(k, l)=1-p(k, l)+\frac{\gamma(1-\bar{p})}{1-\gamma \hat{k}} k
$$

where $\bar{p}=\sum_{l} \bar{H}(l) \sum_{k} P(k) p(k, l)$.

Hereafter we maintain the assumption that $\gamma k^{\max }<1$ and that consumers play the unique equilibrium in the consumption stage. In the first stage, the ability of the monopoly to design targeted pricing strategies depends on the information the monopoly possesses about the network of influence. The benchmark case is when the monopoly knows the distributions of influence, $P$ and $H$, but knows neither the in-degree nor the out-degree of any individual consumer. In such a situation the monopoly charges a uniform price, i.e., $p(k, l)=p$ for all $k, l$. We will compare this benchmark case with three other cases

a. The monopoly knows the out-degree of each consumer and price discriminates on outdegree, i.e., $p(k, l)=p(k)$ for all $l$. 
b. The monopoly knows the in-degree of each consumer and price discriminates on in-degree, i.e., $p(k, l)=p(l)$ for all $k$.

c. The monopoly knows the in-degree and the out-degree of each consumer and price discriminates on both.

In each of these cases the monopoly chooses a price schedule $\{p(k, l)\}_{k, l \in D}$ to maximize its expected profit

$$
\Pi=\sum_{l} \sum_{k} H(l) P(k) x(k, l) p(k, l),
$$

where $x(k, l)$ is the equilibrium consumption decision given by expression (2) and the choice of $p(k, l)$ is restricted depending on the information that the monopoly possesses as described above. The following assumption guarantees that the monopoly's maximization problem is well-behaved and has a unique interior solution (see Proposition 11 in the Appendix). We maintain assumption 1 throughout the paper.

Assumption 1. $\gamma k^{\max }<\frac{1}{2}$.

\subsection{Interpretations of the model}

We briefly discuss the main assumptions of the model and the possible interpretations of the framework.

Our main interpretation of the consumption stage is that when consumers take their consumption decisions, they take into account local network effects, but they have only partial information about their future interactions, i.e., they know the degree distributions and their own (expected) degrees, but not the degrees of their neighbors. This interpretation is consistent with models of network games where there is incomplete information about the network, e.g., Galeotti et al. (2010). An alternative interpretation is that network effects are global and so each consumer cares about the weighted average consumption of the entire population of consumers, but with different intensities, as specified by their particular type $(k, l)$. So, our formulation accounts both for local and global network effects.

An important and novel element of our model is the distinction between the level of influence of a consumer and the susceptibility to influence. The out-degree parametrizes the latter, e.g., how many other consumers does the consumer look up to, listen to, benefit from sharing the use of the good with, etc. The in-degree parametrizes the former, e.g., how many other consumers look up to the consumer, listen to her, benefit from sharing the use of the good with her, etc. As discussed in the introduction, the separation between the level of influence of a consumer and her susceptibility to influence, and possible correlations between these two dimensions, is important because it is empirically relevant in markets (see footnote 1).

In some applications it is more intuitive to think of connections as undirected. That is, a connection may capture a social interaction and the good may be any social interaction good such as Facebook.com or online gaming, or any collaboration tool such as Dropbox.com. In its most basic formulation, undirected influence is captured in our model by perfect correlation between consumers' in- and out-degrees. Moreover, even under the assumption of undirected 
connections our setup allows for a different patterns of influence. For example, the case in which individuals have symmetric interactions, but some are more susceptible to influence than others, is captured by replacing an individual's out-degree $k$ with her "effective out-degree" $\widetilde{k}$, which equals $k$ times the relative susceptibility of that particular individual to be influenced by a single connection. A similar approach could be used to accommodate charismatic individuals who generate more influence in each of their interactions.

The richness of the model allows us to consider a variety of goods and patterns of network effects. To see how, consider goods for which an individual with a high out-degree is less influenced by each one of her contacts - e.g., when deciding how much time to spend in a golf club, having more golf playing friends increases a consumer's demand only up to a point, due to time constraints. We accommodate such network effects by replacing a consumer's outdegree $k$ with a concave function $\lambda(k)$. This transformation covers many cases that received attention in the literature. For example, it has been suggested that in some cases, an individual is influenced by the average consumption of her contacts, or by different moments of the distribution of her contacts' consumptions. This could be the case with goods that have a competitive element, such as online gaming - an individual wants to get to a level of expertise that is at least as high as the lowest/average/highest level of expertise of her contacts, or alternatively, with goods that have comparable alternatives which also exhibit network effect, such as mobile phones brands, and other competing platforms. ${ }^{9}$ Accounting for consumers who are influenced by the average consumption of their contacts, can be done in our formulation by setting the out-degrees of all of the consumers to equal 1 so that ${ }^{10}$

$$
U_{i}\left(x_{i}, \mathbf{x}_{-i}, p_{i}, k\right)=\left(1-p_{i}\right) x_{i}-\frac{1}{2} x_{i}^{2}+\gamma x_{i} A\left(\mathbf{x}_{-i}\right) .
$$

The example of the adoption of mobile phone brands suggests that one may also be interested in considering binary adoption decisions. The following section (section 2.1.1) demonstrates that this paper covers this case as well.

We conclude by remarking that we only investigate linear pricing. This is without loss of generality in the binary adoption version of the model. Otherwise, for different levels of information about in- and out-degrees, the monopolist could propose bundles with specific quantities and price and hope to increase profit (relative to linear pricing). Without information on in-degree and out-degree the problem of the monopolist becomes a screening problem. When the monopolist has detailed information the monopolist will offer specific bundles to consumers based on their in-degree and their out-degree. Given the prevalence of linear pricing in markets, we view our analysis as a useful benchmark; we leave a complete analysis of non-linear pricing for future research.

\footnotetext{
${ }^{9}$ Assuming that all of a consumer's connections have a mobile phone, the consumer wants to adopt the phone which most of her friends adopt. Hence, she looks at the average consumption/adoption rate of her friends.

${ }^{10}$ For consistency, this requires scaling down the in-degrees of all of the consumers by a factor of $\frac{1}{\hat{k}}$.
} 


\subsubsection{Binary product adoption}

The model, analysis, and results can be modified with minor changes to accommodate a discrete good for which consumers make binary choices of whether to adopt or not to adopt. In that case, consumers' strategies will be represented by probabilities of adoptions $\left\{x_{i}\right\}_{i \in N}$ such that $x_{i} \in[0,1]$, and the prices will be interpreted as prices of adopting the good. Formally, each consumer $i$ has a unit demand for the good, and draws an intrinsic value $\theta_{i}$ from the uniform distribution over the unit interval. Intrinsic values are independently distributed. Consumers experience local network externalities in the sense that their value for the good increases by the constant value $\gamma>0$ whenever one of their neighbors adopts the good. That is,

$$
u_{i}\left(x_{i}, \mathbf{x}_{-i}, p_{i}\right)=x_{i}\left(\theta_{i}-p_{i}+\gamma \sum_{j \in N_{i}} x_{j}\right) .
$$

A consumer $i$ adopts the good if and only if $E\left[\theta_{i}-p_{i}+\gamma \sum_{j \in N_{i}} x_{j}\right]>0$, which happens with probability $1-p_{i}+\gamma \sum_{j \in N_{i}} x_{j}$. Therefore, the probability that consumer $i$ adopts the good is $x_{i}=1-p_{i}+\gamma k A\left(\mathbf{x}_{-i}\right)$. This is exactly the first order conditions in the divisible good formulation (when $x_{i}$ is interpreted as a quantity), and all of our analysis goes as is to this adoption framework.

\section{Preliminary results: equilibrium pricing and con- sumption}

In our benchmark case the monopoly has information on the distributions of influence, $P$ and $H$, but has no information on consumers' types and therefore charges a uniform linear price, $p(k, l)=p$ for all $k, l$.

Proposition 2. If the monopoly only knows the distributions $P$ and $H$, then the optimal price is $p=1 / 2$ and does not depend on the properties of the network. The equilibrium consumption level of a consumer increases in the average level of influence and increases linearly in the consumer's out-degree. The expected monopoly profit increases in the average level of influence.

The price charged is independent of the characteristics of the network and therefore the equilibrium demand of a consumer depends only on her out-degree. Since there are network externalities, a consumer's demand is increasing in her out-degree; this effect is linear because of the quadratic payoff specification (i.e., linear best reply). As a consequence, both the average equilibrium consumption and the monopoly profits increase in the average level of influence across consumers. Since the effect of the out-degree on consumption choices is linear and since the price is constant, only the average level of influence matters for monopoly's profits. As we shall see in the next sections, when the monopoly can price discriminate, additional statistics of the networks become relevant. 


\subsection{Price discrimination}

We now describe the properties of optimal price schemes, when the monopoly has additional information about the level of influence of each consumer.

Proposition 3. Whenever the monopoly discriminates on out-degrees (resp. in-degrees), the optimal price schedule depends on the average level of influence and on the variance of the out-degree distribution (resp. in-degree distribution). Furthermore, if the monopoly price discriminates on out-degrees (resp. in-degrees), the price to a consumer linearly increases in her out-degree (resp. linearly decreases in her in-degree).

Out first insight is that when the monopoly price discriminates, consumers who are more susceptible to influence pay a price premium, whereas influential consumers receive a price discount. The size of the discount/premium depends on the patterns of influence, as captured by the average influence and the variance of the out- and in-degree distributions. We illustrate this next.

When the monopoly discriminates only based on out-degrees, the optimal price charged to a consumer with out-degree $k$ is

$$
p(k)=\frac{1}{2}-\frac{\gamma}{2}\left[\frac{\gamma \sigma_{k}^{2}+\hat{k}-k}{4-4 \gamma \hat{k}-\gamma^{2} \sigma_{k}^{2}}\right],
$$

where $\sigma_{k}^{2}$ is the variance of the out-degree distribution. Hence, the price premium paid by a consumer per unit increase in out-degree is

$$
p(k+1)-p(k)=\frac{\gamma}{2\left(4-4 \gamma \hat{k}-\gamma^{2} \sigma_{k}^{2}\right)},
$$

which is higher the higher is the average level of influence and the more dispersed is the distribution of out-degrees in the population.

To see why an increase in the average influence increases the out-degree price premium note that holding an individual consumer's out-degree fixed, an increase in the average level of influence implies that the same consumer is, in expectations, more influential (i.e., has a higher expected in-degree $l$ ). Consequently, the monopoly would like to charge the consumer a lower price. However, this per-unit discount is more costly to apply to a consumer with a higher pre-discount demand, since it applied to the entire quantity.

The intuition for the effect of a change in the variance of out-degrees on the out-degree price premium is related to a very general fact in networks: when the variance of the out-degrees increases, the expected out-degree of an individual at the (out) end of a randomly chosen link also grows. This is true even if the average out-degree remains constant. ${ }^{11}$ As a result,

\footnotetext{
${ }^{11}$ Formally, note that the expected out-degree of an individual at the end of a randomly $\operatorname{chosen} \operatorname{link}$ is $\sum_{k} \bar{P}(k) k=$ $\frac{1}{\hat{k}} \sum_{k} P(k) k^{2}=\frac{\sigma_{k}^{2}}{\hat{k}}+\hat{k}$. Therefore, a mean preserving spread in the distribution of out-degrees increase the expected out-degree of an individual at the out end of a randomly chosen link.
} 
for a consumer with out-degree $k$, increasing the variance of out-degrees in the population increases the probability that a consumer who is connected to her (via her in-links) has a higher out-degree. As a result, it is more cost effective for the monopoly to reduce the price for the consumer than to do so for her neighbor relative to the pre-increase variance. Similarly to the effect of an increase in average influence, this effect is also weaker for consumers with high out-degrees, because they already have high pre-discount demand.

In contrast, when the monopoly discriminates based on in-degrees (see expression 22 in the appendix) then the price discount received by a consumer per unit increase in her in-degree is

$$
p(l)-p(l+1)=\frac{\gamma}{2\left(4-4 \gamma \hat{k}-\gamma^{2} \sigma_{l}^{2}\right)},
$$

which increases in the average level of influence and in the variance of the in-degree distribution, $\sigma_{l}^{2}$. The intuition follows a mirror image of the intuition for the effects for discrimination based on out-degree. These insights carry over to the case where the monopoly discriminates both on out- and in-degree (see expression 23 in appendix). Summarizing:

Corollary 1. If the monopoly price discriminates on out-degrees (resp. in-degrees), the price premium paid (resp. price discount received) by a consumer per unit increase of the out-degree (resp. in-degree) is increasing in the average level of influence and in the variance of the out-degree (resp. in-degree) distribution.

\subsection{Consumption}

The following proposition shows how the equilibrium consumption depends on the outdegrees and in-degrees of consumers, and how these relationships depend on the ability of the monopoly to price discriminate on these levels of influence.

Proposition 4. The equilibrium consumption level of a consumer at the optimal price schedule depends on the average level of influence and on the variance of the out-degree distribution (resp. in-degree distribution) whenever the monopoly can discriminate on out-degrees (resp. indegrees). Furthermore, if the monopoly price discriminates on out-degrees (resp. in-degrees), the equilibrium consumption level of a consumer linearly increases in her out-degree (resp. out-degree and in-degree).

First, when the monopoly only discriminates on out-degrees there are two confounding effects. On the one hand, a consumer with high out-degree pays a higher price and this decreases her consumption; on the other hand a consumer with high out-degree enjoys more externalities and this boosts her consumption. The merit of the proposition is to show that optimal price is set so that more susceptible agents consume more. The intuition follows a similar logic as in the theory of monopoly pricing in environments without network externalities: the reason that high out-degree individuals pay more than low out-degree individuals is that the monopoly does not want to forfeit the high price on the large number of units that they consume. For 
this to hold, it must be the case that the high out-degree consumers consume more than low out-degree ones. ${ }^{12}$ Note also that when the monopoly only discriminates on out-degrees, consumption choices are independent of consumers' in-degrees.

Second, when the monopoly only discriminates on in-degrees, the consumption decision of a consumer depends both on his in-degree and on his out-degree, but in a separable way. First, given any out-degree, agents with high in-degrees receive price discounts, so their consumption is higher than agents with low in-degrees. At the same time, since the price schedule is independent of out-degrees, more susceptible agents consume more due to their higher exposure to network externalities.

Finally, equilibrium consumptions depend also on the variance of the in-degree and outdegree distributions, and on the average level of influence. The variances of the distributions of in- and out-degrees affect consumption only through their effect on the optimal price schedules. For example, if the monopoly price discriminates on out-degrees, an increase in the variance of the out-degree distribution decreases the price that a consumer with a given out-degree $k$ pays and therefore that consumer demands more of the good.

The effect of the average level of influence on equilibrium consumption is more subtle. When the monopoly discriminates only based on out-degrees, the consumption level of a consumer increases in the average level of influence. However, when the monopoly discriminates on in-degrees, the effect of a change in the average level of influence on the consumption of a consumer depends on his out-degree and in-degree. For example, if the monopoly discriminates only based on in-degrees, then the quantity consumed by a consumer with out-degree $k$ and in-degree $l$ is

$$
x(k, l)=1-\frac{2-\gamma \hat{k}-\gamma l}{4-4 \gamma \hat{k}-\gamma^{2} \sigma_{l}^{2}}+\frac{\gamma\left(2 \hat{k}+\gamma \sigma_{l}^{2}\right)}{\hat{k}\left(4-4 \gamma \hat{k}-\gamma^{2} \sigma_{l}^{2}\right)} k,
$$

which increases in the average level of influence if $l$ is sufficiently large, but not necessarily when $l$ is small. The reason lies in the pricing scheme. On the one hand, an increase in the average level of influence leads to higher consumption, keeping constant the price schedule. On the other hand, the price charged to each individual increases in the average level of influence when the monopoly discriminates on in-degrees, and this reduces demand. Such an increase in price is lower for high in-degree individuals. Therefore, only for consumers' with sufficiently high in-degree this price effect is dominated by the externality effect.

\footnotetext{
${ }^{12}$ The classic unit elasticity requirement does not hold due to the network externalities. However, the same logic applies.
} 


\section{Main results: profits, welfare, and the value of information}

\subsection{Profits and the value of network information}

We now describe how the profits of the monopoly depend on the characteristics of the network and on the knowledge of the monopoly about the network.

Proposition 5. For all price discrimination schemes, the profit of the monopoly is increasing and convex in the average level of influence. Furthermore, it is increasing and convex in the variance of the out-degree distribution (reps. in-degree distribution) if the monopoly price discriminates on out-degrees (reps. in-degrees).

Our first insight here is that simple statistics of the network such as the average level of influence and the variance of the out-degree and the in-degree distribution are sufficient to compute the profits that a monopoly would obtain by price discriminating. This information on the network is potentially easy to collect. It can therefore be used as a first step for the monopoly to decide whether it is worth investing in collecting or purchasing precise individual level information on in-degrees and/or out-degrees. Similarly, this information can easily be conveyed by a company seeking to sell the data to the monopoly, without revealing the data itself, thus reducing information asymmetries and facilitating the trade of information.

The second insight captures the effects of changes in the average level of influence and in the variance of the degree distributions on the expected monopoly profits. To see why profits increase in average influence, consider the following intuition. For a given price schedule, an increase in the average influence is synonymous of an increase in network externalities and this triggers higher demand of each consumer and higher monopoly profits. Hence, at the new optimal price schedule, the profit of the monopoly must also increase.

The intuitive reason why, when the monopoly discriminates, say, on out-degrees, the level of profits is increasing in the variance of the out-degree distribution, is that the monopoly uses the information on influence to price discriminate. If the degrees of different consumers are similar, the scope of price discrimination is limited (e.g., if all consumers have the same outdegree, there is no room for discrimination based on out-degree), whereas a high spread allows for significant price discrimination. This implication of Proposition 5 formalizes a recurrent and important theme in marketing, stressing the importance of strategies that take advantage of structural differentiation, see e.g., Krackhardt (1996).

Proposition 5 highlights how the characteristics of the network of influence affect the level of profits under a given knowledge of the monopoly. We now investigate the characteristics of the network of influence that makes the monopoly willing to acquire information on consumers' influence. The value of the monopoly of learning the out-degree (resp. in-degree) relative of noinformation is defined as the difference in the expected profit when the monopoly discriminates on out-degrees (resp. in-degree) and the expected profit under uniform price. We then say that the monopoly prefers to learn the out-degree rather than the in-degree when the value of 
learning the out-degree is higher than the value of learning the in-degree. Corollary 2 answers the following questions: How does the value of learning in-degrees and out-degrees depend on the network of influence? If the monopoly can only acquire information on either the in-degree or the out-degree, which information should the monopoly acquire?

Corollary 2. The value for the monopoly from learning the out-degrees (resp. in-degrees) of all consumers relative to having no information on individual consumers' out- and in-degrees is increasing in the average level of influence and in the variance of the out-degree (resp. indegree) distribution. Moreover, the monopoly prefers to learn individual consumers' out-degrees rather than their in-degrees if and only if the variance of the in-degree distribution is larger than the variance of the out-degree distribution.

Recall that Bearden and Etzel (1982) shows that the level of influence among consumers increases with the "conspicuousness" of the product. Thus, one prediction from Corollary 2 is that a monopoly selling conspicuous goods is more likely to invest in acquiring the information on the in- and/or out-degrees of its consumers.

To see why the value of information on in-degrees or out-degrees is higher when the distributions of the intensities of influence are more dispersed, ${ }^{13}$ recall that the profit of the monopoly under uniform price is independent of the variance of the degree distributions, whereas the profit of the monopoly is increasing in the variance of the degree distributions under price discrimination.

The fact that the value of information on in-degrees or out-degrees of consumers is higher for products with a higher average level of influence is less immediate because in the case of uniform price the monopoly's profits are also increasing in the average level of influence. Therefore, corollary 2 suggests that by price discriminating the monopoly can leverage the increase in the network externalities more effectively.

Finally, whether the monopoly prefers to learn the in-degrees of consumers or their outdegrees depends solely on the variances of the corresponding distributions: the monopoly prefers to learn the degrees that have higher variance, as a high spread allows for significant price discrimination.

One question, which is not answered by Corollary 2, is the following: When will a monopoly invest in learning both the out- and in-degrees of consumers, as opposed to investing only in information on the more valuable of the two? In other words, we would like to know whether the value of information is separable, or whether the data on consumers' out- and in-degrees complement or substitute each other in terms of generating monopoly profit.

Definition 1. We say that the value for the monopoly from learning the out-degrees and in-degrees for all consumers exhibits complementarity if:

$$
\left.\Pi_{\text {in/out }}-\Pi_{\text {out }} \geq \Pi_{\text {in }}-\Pi \quad \text { (or equivalently } \Pi_{\text {in } / \text { out }}-\Pi_{\text {in }} \geq \Pi_{\text {out }}-\Pi\right) .
$$

\footnotetext{
${ }^{13}$ It is also the case that the value for the monopoly of learning the in-degrees and out-degrees relative of noinformation is increasing in the variance of the in-degree distribution and in the variance of the out-degree distribution.
} 
If the inequalities are reversed we say that the value for the monopoly from learning the out-degrees and in-degrees of all consumers exhibits substitutability.

Corollary 3. The value for the monopoly from learning the out-degrees and in-degrees of all consumers exhibits complementarity.

Corollary 3 implies that the monopoly values having data on individual consumers' indegrees more if the monopoly already has data on individual consumers out-degrees and viceversa. An immediate implication is that if the variances of the in- and out-degree distributions are equal (which implies that the values of learning the in-degree or out-degree are equal), and if the cost of acquiring each of the different data is the same, then a monopoly will either invest in collecting data on both individual consumers in- and out-degrees or on neither.

\subsection{Consumer surplus and aggregate welfare}

We conclude this section by considering the implication of targeted pricing strategies on consumer surplus and aggregate welfare. We are particularly interested in whether moving from a uniform pricing strategy to a pricing strategy that incorporates information on indegrees or out-degrees leads to an increase or rather a decrease in consumer surplus. ${ }^{14}$ This exercise provides some guidance to competition authorities that may be concerned with the effects on consumer surplus and welfare of allowing firms to use information about consumers' network of influence in product markets.

Recall that for a given consumption profile $\left(x_{i}, \mathbf{x}_{-i}\right)$ and price $p_{i}$, the expected utility to consumer $i$ with out-degree $k$ and in-degree $l$ is

$$
U_{i}\left(x_{i}, \mathbf{x}_{-i}, p_{i}, k\right)=\left(1-p_{i}\right) x_{i}-\frac{1}{2} x_{i}^{2}+\gamma x_{i} k A\left(\mathbf{x}_{-i}\right) .
$$

In a Nash equilibrium of the consumption stage, consumer $i$ plays a best reply; that is, $i$ 's consumption $x_{i}^{*}$ satisfies

$$
\left(1-p_{i}\right)-x_{i}^{*}+\gamma k A\left(\mathbf{x}_{-i}^{*}\right)=0
$$

We can then rewrite the equilibrium utility of consumer $i$ as follows

$$
U_{i}^{*}=\frac{1}{2}\left[x_{i}^{*}\right]^{2}
$$

Therefore, to determine whether a consumer with out-degree $k$ and in-degree $l$ is better off when the seller does not discriminate as compared to the case where, say, the seller discriminates on out-degree, we simply need to compare the equilibrium consumption of the consumer in the two regimes. Furthermore, aggregating consumers' utilities across different types we obtain a simple expression for consumer surplus. By letting $x(k, l)$ be the consumption of a consumer

\footnotetext{
${ }^{14}$ For the sake of brevity we do not compare the case of no price discrimination with the case of price discrimination on both in-degrees and out-degrees, but analogous results and intuitions apply to that case.
} 
$(k, l)$, consumer surplus is

$$
C S=\frac{1}{2} \sum_{l} \sum_{k} H(l) P(k)\left[x^{*}(k, l)\right]^{2}
$$

\section{Proposition 6.}

1. Suppose we move from no-discrimination to discrimination on out-degrees. There exists a threshold $k^{\prime}>\hat{k}$ so that a consumer is better off if, and only if, her out-degree $k \leq k^{\prime}$. Furthermore, for every $\gamma$ and $k^{\max }$, if the variance of the out-degree distribution is high enough then $k^{\prime}>k^{\max }$.

2. Suppose we move from no-discrimination to discrimination on in-degrees. There exists a threshold function $l^{\prime}(k)$, which is decreasing in $k$, so that a consumer with out-degree $k$ and in-degree $l$ is better off if, and only if, her in-degree $l \geq l^{\prime}(k)$.

Price discrimination of any form increases average consumption, which has a positive effect on the utility of each of the consumers. On the other hand, when the seller discriminates on out-degrees, consumers who are more susceptible to influence pay higher prices than consumers with low out-degrees. It is then not surprising that consumers with low out-degrees benefit from the introduction of such price discrimination. The proposition points out, however, that when the out-degrees are very dispersed, also consumers with high out-degrees benefit from the introduction to price discrimination. To see why, note that when the variance of the out-degree distribution is high, targeted pricing strategies are more effective in boosting average consumption, and this gives incentives to high out-degree consumers to increase their consumption, despite paying higher prices. Furthermore, note that part 1 of proposition 6 implies that, when the variance of the out-degree distribution is high, and we move from nodiscrimination to price discrimination on out-degrees, then all consumers are better off. As the seller always benefits from price discrimination, price discrimination on out-degrees leads, in this case, to a Pareto improvement.

When discrimination is on in-degrees, influential agents receive price discounts. So, within consumers with a certain out-degree, more influential consumers benefit from price discrimination on in-degrees. Moreover, the fact that the function $l^{\prime}(k)$ is decreasing in $k$ implies that consumers with a high out-degree may benefit from discrimination on in-degrees even if they are not as much influential as some consumers with a low out-degree. The reason is that by price discriminating the seller stimulates overall consumption and therefore, fixing the in-degree of a consumer, consumers with high out-degree enjoy greater consumption externalities. In general, the introduction of in-degrees price discrimination may be beneficial for some consumers but not for others. However, we can prove an unambiguous result for the aggregate consumer surplus.

Proposition 7. Consumer surplus increases when we move from no-discrimination to discrimination on in-degrees.

That discrimination on in-degrees increases consumer surplus is interesting as there are many examples of discrimination on the degree of influence of consumers. Examples include 
conspicuous consumption goods and fashion goods, as well as social interaction goods. Such examples often take the form of seeding, which is equivalent to selling a good for a price of zero to selected influential consumers, or even paying such influential consumer to use the good, which is equivalent to setting negative prices for high in-degree individuals.

To conclude our discussion of welfare, we note that the additional profit for the monopoly from introducing any type of price discrimination is greater than any loss to the consumer surplus. That is, aggregate welfare increases when we move from no discrimination to discrimination on in-degrees or out-degrees.

\section{Correlation between in-degree and out-degree}

So far we focused on the case that at the individual consumer's level, in-degrees and outdegrees are independent. Nevertheless, as we reviewed in the introduction, correlations between the in-degrees and out-degrees of individuals have been documented in several studies that study peer influence in consumption decisions. It is also natural when thinking of different agents in the society, for example, celebrities influence many people but they are not always influenced by many people. Our results described in the previous section can be extended to the case of correlation. In fact, in section 6 when we evaluate the value of information in real social networks, we use the derivation of optimal profit obtained for general correlations structure, available in the appendix.

In this section we aim to answer the new questions raised by considering correlation between the in- and out-degrees of consumers: Is the expected profit of the monopoly higher or lower when the big influencers in a market are not influenced much by others? How does the willingness of the monopoly to invest in gathering information depend on whether the more influenced consumers also influence others? To this end, we now examine the impact that a change in correlation between consumers' in-degrees and out-degrees has on the monopoly's profits and the value of information.

Let $P(k \mid l)$ be the probability that a node with in-degree $l$ has out-degree $k$, and let $H(l \mid k)$ be the probability that a node with out-degree $k$ has in-degree $l$. By definition, the following consistency conditions apply: for all $l$ and $k, \sum_{l} P(k \mid l) H(l)=P(k)$ and $\sum_{k} H(l \mid k) P(k)=$ $H(l)$. For simplicity, we assume in this section that the variance of the in-degree and the variance of the out-degree are the same, i.e., $\sigma_{k}^{2}=\sigma_{l}^{2}=\sigma^{2}$. Therefore, the correlation coefficient can be written as

$$
\rho=\frac{\operatorname{Cov}(k, l)}{\sigma^{2}}=\frac{E[(k-\hat{k})(l-\hat{k})]}{\sigma^{2}}=\frac{E[k l]-\hat{k}^{2}}{\sigma^{2}} .
$$

The case of perfect positive correlation occurs when $H \equiv P$ and $\rho=1$. The case where there is no correlation obtains when $\rho=0$. When the monopoly price discriminates on in-degree (resp. out-degree), it turns out that in determining the optimal price schedule the monopoly will take into account the average out-degree (resp. in-degree) of a consumer conditional on

the consumer's in-degree (resp. out-degree), i.e., $\hat{k}(l)=\sum_{k} P(k \mid l) k$, and the variance of this 
random variable, $\sigma_{\hat{k}(l)}^{2}=\sum_{l} H(l)[\hat{k}-\hat{k}(l)]^{2}$ (resp. $\hat{l}(k)$ and $\left.\sigma_{\hat{l}(k)}^{2}\right)$. Under independence, for any in-degree $l$ it is the case that $\hat{k}(l)=\hat{k}$ and $\sigma_{\hat{k}(l)}^{2}=0$; but for general correlations there is no specific relationship between $\hat{k}(l)$ and $\sigma_{\hat{k}(l)}^{2}$, and the first and second moments of the distribution of influence in the network. The following assumption provides some structure to the relationship between these conditional random variables and the moments of the distribution of influence, which allows us to derive a cleaner characterization.

Assumption 2. The correlation between the in-degree and the out-degree is such that their conditional expectations are linear, i.e., $\hat{k}(l)=E[k \mid l]$ is linear in $l$ and $\hat{l}(k)=E[l \mid k]$ is linear in $k$.

Assumption 2 does not restrict correlation from being negative or positive and $\rho$ can take any value in the range $[-1,1]$ as before. However, the assumption implies that $\hat{k}(l)=\hat{k}+\rho(l-$ $\hat{k}), \hat{l}(k)=\hat{k}+\rho(k-\hat{k})$, and $\sigma_{\hat{l}(k)}^{2}=\sigma_{\hat{k}(l)}^{2}=\rho^{2} \sigma^{2}$.

In what follows, we focus on describing results on how correlation affects optimal profit, and how correlation affects the value of network information for the monopoly.

Proposition 8. Suppose Assumption 2 holds. Regardless of whether the monopoly discriminates based on in-degree, out-degree, both, or none, the monopoly's profit increases in $\rho$.

Recall that agents with high out-degrees have higher demands, ceteris paribus, because of the larger adoption externalities they are exposed to. When the correlation between outdegree and in-degree increases, this means that these high demand consumers are also likely to be very influential, and this has a positive feedback to the consumption decisions of other consumers. This is why the monopoly profits are increasing in the correlation parameter $\rho$, regardless of the sophistication of the price schedule.

We now consider how the value of information depends on the correlation between indegrees and out-degrees. That is, how does the degree correlation affect the value for the monopoly of learning individual consumers' degrees? How does the degree correlation affect possible complementarity or substitutability in the value for the monopoly from learning the indegrees and out-degrees of consumers? Before providing the result we note that our assumption that the variance of the out-degree distribution and the variance of the in-degree distribution are the same, implies that the expected profit of the monopoly, when it can discriminate either on in-degree or on out-degree (but not on both), is the same. This is true for any level of correlation (see appendix). Hence, the value of information is convex if and only if information on out-degrees and information on in-degrees are complements.

Proposition 9. Suppose assumption 2 holds. The value for the monopoly of learning either the out-degrees or the in-degrees relative of no-information is decreasing in $\rho$. Furthermore, there exists a $\rho^{*} \in(-1,0)$ such that the value for the monopoly from learning the out-degrees and in-degrees of all consumers exhibits substitutability for every $\rho<\rho^{*}$, and complementarity otherwise. 
To get a sense of the implications of Proposition 9, consider as an example Aral and Walker (2012), who study the adoption of an application in Facebook.com. They show that in their data, influence and susceptibility to influence are negatively correlated. Proposition 9 then implies that the negative correlation reduces the price a firm selling such an application will be willing to pay Facebook.com for its network data. Moreover, the negative correlation suggests that information on influence and susceptibility to influence are substitutes (or weak complements), and that it may make sense for a firm selling this type of application in Facebook to invest in learning only one of the distributions (assuming equal costs of acquiring each distribution).

To understand the intuition behind the first part of Proposition 9, note that effective price discrimination entails price premia to consumers with high out-degrees, and price discounts to consumers with high in-degree. Increased correlation between in-degree and out-degree reduces the ability to do that and thus reduces the profitability of price discrimination, which in turn reduces the value of information.

The intuition for the second part of Proposition 9 is more subtle. On the one hand, when

$\rho^{2}$ is large (due to higher or lower correlation), a consumer's out-degrees is a more accurate (i.e., less noisy) predictor of her in-degree and vice-versa. As a result, an increase in $\rho^{2}$ reduces the complementarity between information on an individual's in-degree and her out-degree. On the other hand, when in-degrees and out-degrees are positively correlated, information only on a consumer's out-degree (resp. in-degree) is not particularly useful for price discrimination. This follows the same logic as the intuition for the first part of Proposition 9. At the same time, information on both in-degrees and out-degrees allows the monopoly to detect consumers with high out-degrees (resp. in-degrees) and low in-degrees (resp. out-degrees), which allows the monopoly to price discriminate even in the presence of high positive correlation between in-degrees and out-degrees.

\section{The value of information on social, financial, and advice networks}

We provide a simple exercise to illustrate the potential use of targeted pricing strategies across different products. We consider a dataset that includes networks covering three types of relationships between individuals in 75 villages in rural India: social, advice and financial. Our premise is that these three types of relationships capture the patterns of externalities for different products, and we focus on comparing, across these three networks, the value of information, the level of complementarity of information, and the consumer surplus.

\subsection{Description of the data}

The data are from 75 rural villages in Karnataka, an area of southern India within a few hours of Bangalore. The average population per village is 926.48. The survey was designed as 
part of a study of the deployment of a microfinance program (see Banerjee et al. 2011, and Jackson et al 2012). We refer to these papers for a detailed discussion of the data set and its limitations.

Only one-half of the households were surveyed, which could bias (most likely downward) the value of information which we compute. Households were selected by a stratified random sample in order to control for selection biases, with households stratified by religion (Hindu, Muslim, Christian) and also by geographic sub-locations based on a full census of the villages that was conducted just prior to and in conjunction with the survey. Each surveyed individual was asked to name the people with whom he or she has various sorts of relationships.

We make use of the answers to a subset of the questions asked to build three networks capturing three different types of interactions: (i) Social: we say that there is a directed social connection from consumer i to consumer $\mathrm{j}$ if consumer i reports visiting $\mathrm{j}$ 's house in her free time; (ii) Advice: we say that there is a directed advice connection from consumer $\mathrm{i}$ to consumer $\mathrm{j}$ if consumer $\mathrm{i}$ reports coming to $\mathrm{j}$ for advice on difficult personal decisions; (iii) Financial: we say that there is a directed financial connection from consumer $\mathrm{i}$ to consumer $\mathrm{j}$ if consumer i names $\mathrm{j}$ as the person to whom she would turn if she needed to borrow kerosene, rice, or money.

The social network proxies influence in the consumption of social goods, communication technologies, games, or even entertainment. The ties in the advice network are based on elements of expertise in certain dimensions. It is then reasonable to expect that an individual consumes more goods if these are used by experts, or people whose judgement the individual respects. Such people would be able to continue providing advice on the use of such goods, ranging from new crops or fertilizers, to medical or health related equipment and services. Financial relationships influence consumption of financial instruments, such as bank accounts (it is often cheaper to transfer money between customers of the same bank), or even working with the same creditors, so that value could be transferred without money changing hands. ${ }^{15}$

The three networks are quite different from each other. The advice network is the most sparse, with average degree of 1.07, whereas the social and financial networks are denser with average degrees of 1.52 and 1.83 respectively. The high density of the financial network suggests that the society in the surveyed villages relies heavily on risk-sharing and informal financial arrangements. The financial network has also the largest variances of its in- and out-degrees distributions, which equal 5.28 and 1.48, respectively. This is compared with variances of 3.44 and 1.04 for the in-degree and out-degree distributions in the social network, and 3.29 and 0.6 for the corresponding variances in the advice network. The degree correlation is positive in all three networks, but takes significantly different values. Ranging from 0.08 in the advice network, to 0.2 and 0.22 in the financial and social networks respectively. The difference in degree correlation is not surprising: advice is based on expertise, so experts are expected to have high in-degrees, but there is no reason to expect that an expert will need more advice than a non expert. On the other hand, social networks and lending networks capture more

\footnotetext{
${ }^{15}$ For example, an individual can vouch for another individual, or commit to pay a debt if left unpaid by the borrower. See also Karlan et al. (2009).
} 
reciprocal relationships.

The aforementioned measures are for the networks which include all villages. However, individuals from different villages are rarely connected, suggesting that it is realistic to assume that individuals form their beliefs on the patterns of interactions in their environment based on the village-level degree distributions and correlations. Our framework is sufficiently rich to accommodate this local structure - we simply model the monopoly's decision as a set of independent maximization problems, one for each village.

Finally, the networks in our data do not exhibit equal variances of the in- and out- degree distributions, nor do they satisfy assumption 2 (linearity of conditional expectations). We therefore use our general formulation from Appendix A to analyze the data. In that sense, our results also demonstrate the robustness of our analytical results in the previous sections.

\subsection{The value of network information}

Our theoretical analysis shows that the value of information is increasing in the variances of the distributions of in- and out-degrees in the population, and in the average level of influence, and decreasing in the correlation between in- and out-degrees of consumers. Figure 1 captures these patterns for a monopoly which prices optimally based on consumers' in- and out-degrees, and assuming that the level of influence over a link is $\gamma=0.007$. Varying $\gamma$ does not qualitatively change the results.

The value of network information is especially high in the financial network, due to the high average degree and high variances. In contrast, the advice network, and to some extent the social network, have low average degrees and low variances of in- and out-degrees in most villages, which leads to low values of network information. We note an important difference between the social and the advice network. In the former the variance of the in-degree distribution is similar across villages, while in the latter is quite different (the variance of this value across villages is 5.7 in the advice network, compared with 1.28 in the social network). In other words, the distributions of expertise are very different across villages, whereas the distribution of popularity is quite consistent. This explains the departures from the patterns in the plots of the value of information as a function of the average level of influence, the variance of the out-degree distribution, and the degree correlation when it comes to the advice network.

Our theoretical analysis also relates the degree correlation to whether information exhibits complementarity or substitutability. Proposition 9 suggests that there is a threshold value of the correlation level such that informations exhibits complementarity if the correlation is above the threshold and substitutability otherwise. Figure 2 suggests that the relationship between the degree correlation and the level of complementarity of the information (as captured by $\left.\Pi_{\text {in } / \text { out }}-\Pi_{\text {out }}-\Pi_{\text {in }}+\Pi\right)$ is continuous. Moreover, since the financial and social networks exhibit, on average, higher levels of degree correlation, relative to the advice network, the prediction of our model is that in the social and financial networks a monopoly is more likely to either invest in learning both the in- and out-degrees of all consumers, or none. Whereas in the advice networks, where the degree correlation is low and the variance of the in-degree 

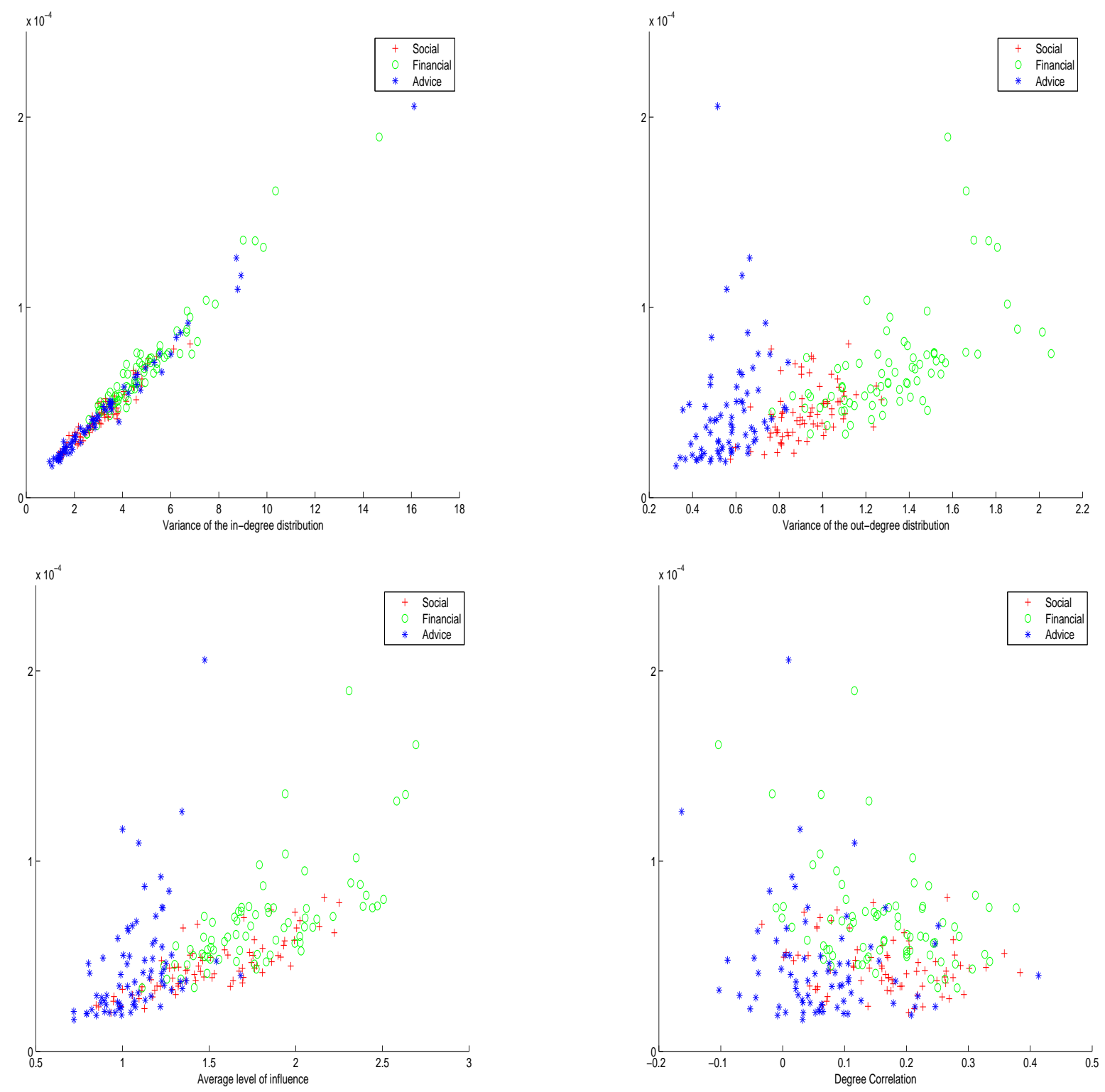

Figure 1 - The value of information on in-degree and out-degrees.

distribution is much higher than the variance of the out-degree distribution we would predict that a monopoly only invest in learning the in-degrees (i.e., detecting the experts), rather than learning the susceptibility to influence of consumers.

Finally, Proposition 6 finds that if the variance of the out-degree distribution is sufficiently large, then all consumers prefer discrimination on out-degree to uniform pricing. We find that in our data the variance of the out-degree is too low to make all consumers better off with discrimination on out-degree. However, we do find a systematic relationship between the variance of the out-degree distribution and the fraction of the consumers who are better off with discrimination on out-degree. Figure 3 demonstrates that the high variance of the out-degree distribution in the financial networks implies that a large majority of consumers prefer discrimination on out-degree to uniform pricing. This is not the case in the social and 


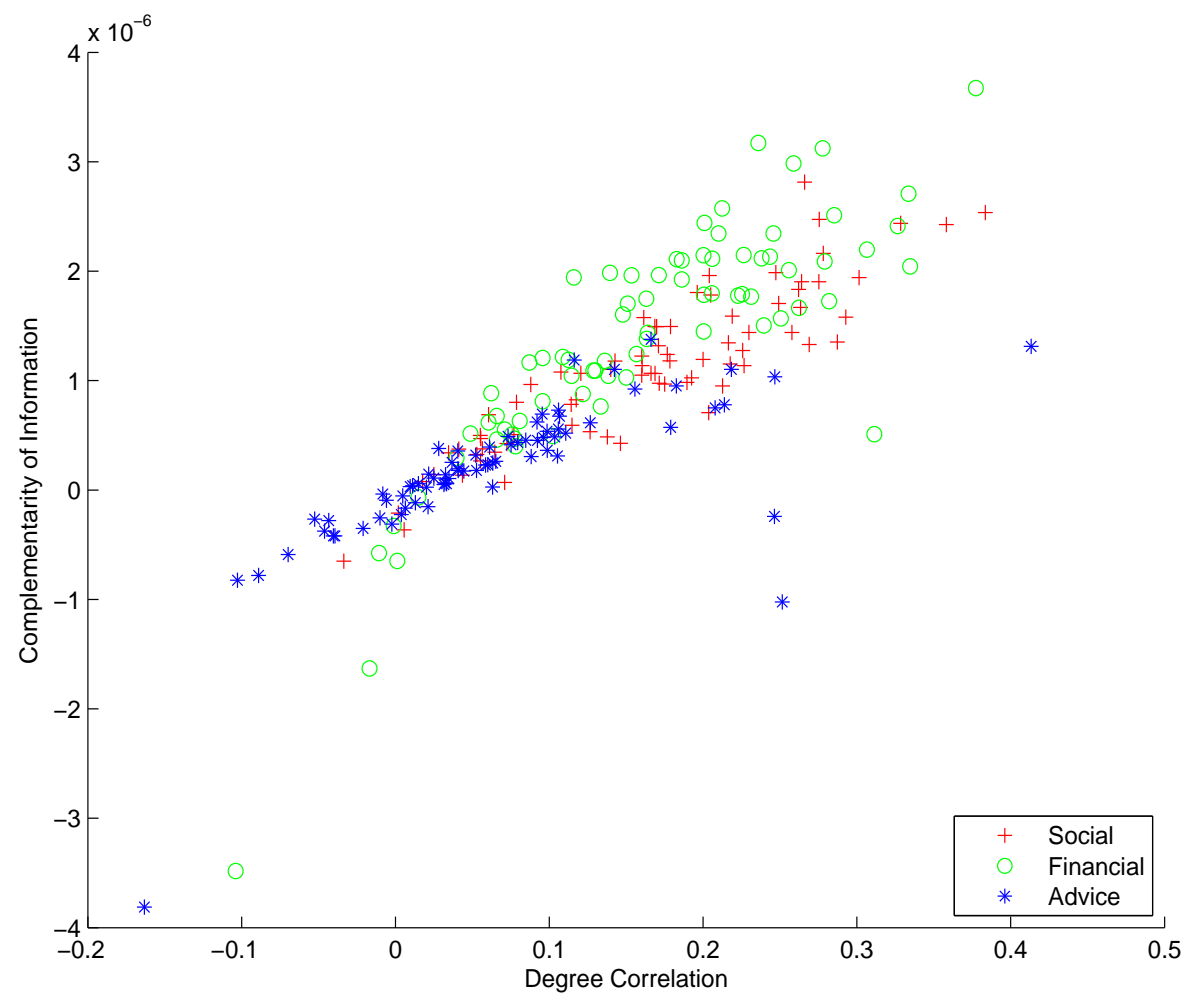

Figure 2 - Complementarities of in-degree and out-degree.

advice networks.

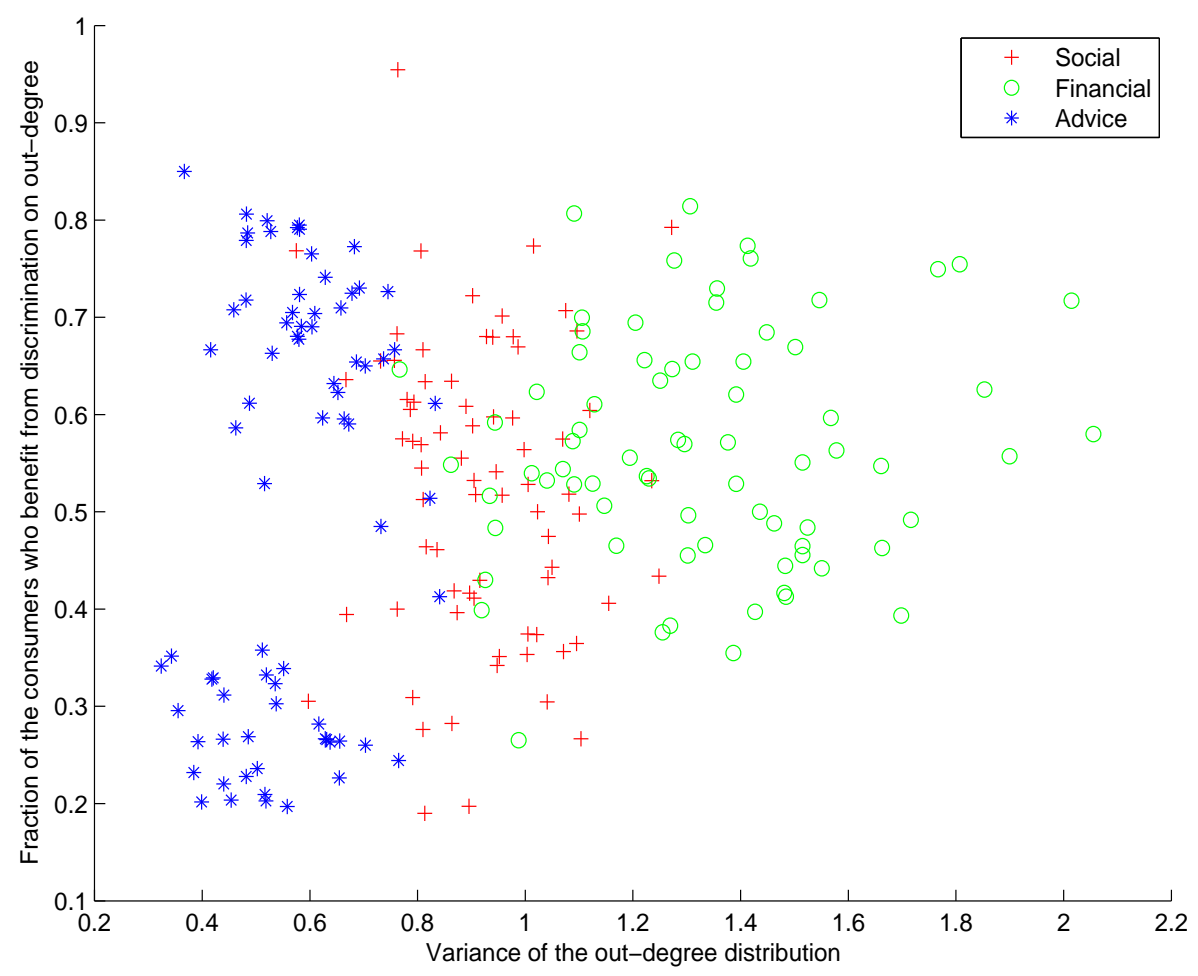

Figure 3 - Price Discrimination and Consumer Surplus. 


\section{Discussion}

Online commerce provides some level of anonymity to consumers with respect to their demographics. However, when combined with records of other online activities, online shopping generates data on local networks externalities that were not available earlier. This example highlights a change in the type of information firms may be able to gather about their consumers. Of the array of important economic questions raised by this shift, this works focuses on the value of network information for a price discriminating monopoly and its effect on consumer surplus.

The principal innovation of this paper is the combination of two important ideas: First, consumers have incomplete knowledge of the network structure - we focus on a simple aspect of the network, the number of personal connections. This formulation allows us to develop a general framework which is tractable. Second, network effects are not necessarily symmetric, some people are more influential, whereas some are more susceptible to influence. We capture this idea by modelling influence as directed. Allowing links to be directed is key in studying the value of information in networks of influence for different products.

The analysis yields several powerful and intuitively appealing insights. We show that simple statistics of the network of influence induced by different products, are sufficient to evaluate the value of information to discriminating monopolies that sell the products. This provides a rigorous guide for monopolies that consider investing in collection or purchase of network data. We also provide a precise measure of the welfare effects of price discrimination that can be used by regulators based on accurate information on several summary statistics of the network.

Finally, this paper focuses on positive network effects, i.e., consumption decisions are strategic complements. This is an important setup that raised significant interest in many disciplines. Nevertheless, we also note that our analysis and solution go through without change for the case of negative network effects, where consumption decisions are strategic substitutes, as long as the negative influence is not too strong. 


\section{References}

1. Aral, S. and D. Walker, 2012, Identifying influential and susceptible members of social networks. Science, 337(6092):337-341.

2. Arthur, D., R. Motwani, A. Sharma, and Y. Xu, 2009, Pricing Strategies for Viral Marketing on Social Networks. Mimeo, Department of Computer Science, Stanford University.

3. Ballester, C., A. Calvo-Armengol, and Y. Zenou, 2006, Who's Who in Networks. Wanted: The Key Player. Econometrica, 74, 1403-17.

4. Banerjee A., A. Chandrasekhar, E. Duflo and M. O. Jackson, 2011, The Diffusion of Microfinance. Working paper MIT and Stanford University.

5. Bearden, W.O. and M.J. Etzel, 1982, Reference group influence on product and brand purchase decisions, Journal of Consumer Research, 9:183-94.

6. Bloch, F. and N. Quérou, 2013, Pricing in social networks. Games and Economic Behavior, 80, 263-281.

7. Bramoullé, Y. , R. Kranton and M. D'Amours, 2011, Strategic Interaction and Networks, American Economic Review. Forthcoming.

8. Campbell, A., 2013, Word of Mouth and Percolation in Social Networks. American Economic Review. Forthcoming.

9. Candogan, O., K. Bimpikis and A. Ozdaglar, 2012, Optimal Pricing in Networks with Externalities. Operations Research.

10. Domingos, P. and M. Richardson, 2001, Mining the network value of customers. Proceedings of the 7th Conference on Knowledge Discovery and Data Mining, 57-66.

11. Farrell, J. and G. Saloner, 1985, Standardization, Compatibility and Innovation. RAND Journal of Economics, 16, 70-83.

12. Feige, U., A Fiat, and A. Shamir, 1988, Zero-Knowledge Proofs of Identity. Journal of Cryptology, 1, 77-94.

13. M. Froomkin, 2000, The Death of Privacy?. Standard Lw Review, 52(5):1461-1543.

14. Galeotti, A. and S. Goyal, 2009, Influencing the influencers: A theory of strategic diffusion. RAND Journal of Economics, 40(3):509-532.

15. Galeotti, A., S. Goyal, M.O. Jackson, F. Vega-Redondo and L. Yariv, 2009, Network Games. Review of Economic Studies.

16. Goyal, S. and M. Kearns, 2012, Competitive Contagion in Networks. STOC.

17. Goldenberg, J., S. Han, D. Lehmann and J. Hong, 2009, The role of hubs in the adoption process. Journal of Marketing, 73(2):1-13.

18. Green, H., 2008, Google: Harnessing the Power of Cliques. BusinessWeek, October 6, 50.

19. Hartline, J., V. Mirrokni and M. Sundarajan, 2008, Optimal Marketing Strategies over Social Networks. Proceedings of WWW 2008: Beijing, China, 189-198.

20. Jackson, M.O., T. Rodriguez-Barraquer and X. Tan, 2012, Social Capital and Social Quilts: Network Patterns of Favor Exchange. American Economic Review. 
21. Karlan D., M. Mobius, T. Rosenblat and A. Szeidl, 2009, Trust and Social Collateral. Quarterly Journal of Economics

22. Katz, M. and C. Shapiro, 1985, Network Externalities, Competition and Compatibility. American Economic Review, 75, 424-440.

23. Keller, E., B. Fay and J. Berry, 2007, Leading the Conversation: Influencers' Impact on Word of Mouth and the Brand Conversation. The Keller Fay Group, Word of Mouth Marketing Research Symposium.

24. Kempe, D., J. Kleinberg and E. Tardos, 2003, Maximizing the Spread of Influence through a Social Network, Proceedings of the 9th Inter-national Conference on Knowledge Discovery and Data Mining, 137-146.

25. Krackhardt, D., 1996, Structural Leverage in Marketing. In Dawn Iacobucci (Ed.) Networks in Marketing, Sage, Thousand Oacks CA. pp. 50-59.

26. Leskovec J., L.A. Adamic and B.A. Hubermann, 2007, The dynamics of viral marketing. ACM Transactions on the Web 1(1):article 5.

27. Nair H.S., P. Manchanda and T. Bhatia, 2010, Asymmetric social interactions in physician prescription behavior: The role of opinion leaders. Journal of Marketing Research, 47(5):883- 895 .

28. N. Newman, 2013, The cost of lost privacy: search, antitrust and the economics of the control of user data. SSRN Working paper.

29. Probst, F, L. Grosswiele and R. Pfleger, 2013, Who will lead and who will follow: Identifying Influential Users in Online Social Networks, Business and Information Systems Engineering, Volume 5, Issue 3, pp 179-193.

30. Sundarajan, A., 2006, Local Network Effects and Network Structure. Mimeo, Stern School of Business, New York University.

31. Tucker C., 2008, Identifying Formal and Informal Influence in Technology Adoption with Network Externalities, Management Science, Vol. 55 No. 12, 2024-2039. 


\section{Appendix}

The Appendix is divided in two parts. In Appendix A we derive results for the general model with correlations between in-degree and out-degree (without Assumption 2). Appendix B proves the results for the case of no-correlation (Proposition 1-7) and the case of correlation under Assumption 2 (Proposition 8-9).

Appendix A. In the general case, the correlation between in- and out-degrees is captured by

$$
\rho=\frac{\operatorname{Cov}(k, l)}{\sigma_{k} \sigma_{l}}=\frac{E[(k-\hat{k})(l-\hat{k})]}{\sigma_{k} \sigma_{l}}=\frac{E[k l]-\hat{k}^{2}}{\sigma_{k} \sigma_{l}} .
$$

Define

$$
\bar{p} \equiv \sum_{l} \bar{H}(l) \sum_{k} P(k \mid l) p(k, l)
$$

and note that

$$
\bar{k} \equiv \sum_{l} \bar{H}(l) \sum_{k} P(k \mid l) k=\frac{1}{\hat{k}} \sum_{l} \sum_{k} H(l) P(k \mid l) k l=\frac{E[k l]}{\hat{k}}
$$

and rewrite $\rho$ as follows

$$
\rho=\frac{\bar{k}-\hat{k}}{\sigma_{k} \sigma_{l}} \hat{k}
$$

The case of perfect positive correlation occurs when $H \equiv P$ and $\rho=1$, which implies that $\sigma=\sigma_{k}=\sigma_{l}$ and $\bar{k}=\frac{\sigma^{2}+\hat{k}^{2}}{\hat{k}}$. The case where there is no correlation obtains when $\rho=0$, or, equivalently, $\bar{k}=\hat{k}$. We start by characterizing the demands of the consumers for any given price schedule set by the monopoly.

Proposition 10. Let $\mathbf{p}$ be any price schedule set by the monopoly in the first stage of the game and specifying for each consumer $i$ a price per unit $p_{i} \in \mathbb{R}$.

1. If $\gamma k^{\max }<1$, then, in the consumption stage, there exists a unique Bayes-Nash equilibrium $\mathrm{x}^{*}$.

2. Now assume that according to $p$ any two consumers with identical in-and out-degrees are priced the same. Then, in the unique equilibrium of the consumption stage any two consumers with identical in- and out-degrees make the same consumption decision. In particular, let $p(k, l)$ and $x(k, l)$ be the price and the demand of a consumer with outdegree $k$ and in-degree $l$. Then, in the unique equilibrium of the consumption stage

$$
x(k, l)=1-p(k, l)+\frac{\gamma(1-\bar{p})}{1-\gamma \bar{k}} k .
$$

Proof of Proposition 10. Existence: Recall that $u_{i}\left(k, x_{i}, x_{-i}\right)=x_{i}-\frac{1}{2} x_{i}^{2}+\gamma k x_{i} A$. Then, adapting Proposition 1 from Glaeser and Scheinkman (2000) to our setup we get that there exists at least one consumption equilibrium if $\forall_{k \in \mathbb{Z}^{+}, p \in \mathbb{R}} \exists_{\bar{x} \geq 0} \forall_{x \leq \bar{x}} \frac{\partial u}{\partial x_{i}}\left(k, \bar{x},(x)_{j \neq i}\right)-p \leq 0$. To see that this condition holds if $\gamma k^{\max }<1$ it is sufficient to note that $\forall_{k \in \mathbb{Z}^{+}, p \in \mathbb{R}} \exists_{\bar{x} \geq 0} \forall_{x \leq \bar{x}} \frac{\partial u}{\partial x_{i}}\left(k, \bar{x},(x)_{j \neq i}\right)-$ 
$p \leq \frac{\partial u}{\partial x_{i}}\left(k^{\max }, \bar{x},(\bar{x})_{j \neq i}\right)-p=1-\bar{x}+\gamma k \bar{x}-p$ and that $\forall_{p \in \mathbb{R}} \exists_{\bar{x} \geq 0} 1-\bar{x}+\gamma k \bar{x} \leq 0$ if and only if $\gamma k^{\max } \leq 1$.

Uniqueness: Proposition 3 in Glaeser and Scheinkman (2000) implies that there exists at most one consumption equilibrium if $\forall_{i}\left|\frac{\partial^{2} u_{i}}{\partial x_{i} \partial A} / \frac{\partial^{2} u_{i}}{\left(\partial x_{i}\right)^{2}}\right|<1$ or $\gamma k^{\max }<1$.

Finally, in an interior equilibrium, for each type $(k, l)$

$$
x(k, l)=1-p(k, l)+\gamma k A,
$$

and therefore

$$
A=\sum_{l} \bar{H}(l) \sum_{k} P(k \mid l) x(k, l)=1-\bar{p}+\gamma \bar{k} A,
$$

or

$$
A=\frac{1-\bar{p}}{1-\gamma \bar{k}}
$$

and plugging back to the expression for $x(k, l)$ we obtain an explicit expression for the equilibrium consumption of type $(k, l)$.

In the remaining of Appendix A, we derive the optimal monopoly prices and the corresponding profits under general correlation structure and different information that the monopoly may have. We begin by assuming that the monopoly's optimization problem is well behaved and derive the corresponding prices, demands, and monopoly profit for price discrimination regime. We follow with an analysis of the conditions that guarantee that the monopoly's optimization problem is, in fact, well behaved.

Uniform price. Let $p(k, l)=p$ for all $(k, l)$. Then $\bar{p}=\sum_{l} \bar{H}(l) \sum_{k} P(k \mid l) p(k)=p$, and

$$
x(k, l)=x(k)=1-p+\frac{\gamma(1-p)}{1-\gamma \bar{k}} k .
$$

The monopoly maximizes

$$
\Pi_{\text {uniform }}=\sum_{k} P(k) p x(k)=p(1-p)\left[1+\frac{\gamma \hat{k}}{1-\gamma \bar{k}}\right]
$$

so, $p^{*}=1 / 2$,

$$
x^{*}(k)=\frac{1}{2}\left[1+\frac{\gamma}{1-\gamma \bar{k}} k\right]
$$

and

$$
\Pi_{\text {uniform }}^{*}=\frac{1}{4}\left[1+\frac{\gamma \hat{k}}{1-\gamma \bar{k}}\right] .
$$

Price discrimination. When the monopoly price discriminates on in-degree (resp. outdegree), it turns out that in determining the optimal price schedule the monopoly will take into account the average out-degree (resp. in-degree) of a consumer conditional on the consumer's in-degree (resp. out-degree), i.e., $\hat{k}(l)=\sum_{k} P(k \mid l) k$, and the variance of this random variable, $\sigma_{\hat{k}(l)}^{2}=\sum_{l} H(l)[\hat{k}-\hat{k}(l)]^{2}\left(\operatorname{resp} . \hat{l}(k)\right.$ and $\left.\sigma_{\hat{l}(k)}^{2}\right)$. 
Discrimination on out-degree. Let $p(k, l)=p(k)$ for all $l$, and so

$$
x(k, l)=x(k)=1-p(k)+\frac{\gamma(1-\bar{p})}{1-\gamma \bar{k}} k,
$$

where $\bar{p}=\sum_{l} \bar{H}(l) \sum_{k} P(k \mid l) p(k)$. The monopoly chooses $p(k)$ for all $k$, to maximize $\Pi_{\text {out }}=$ $\sum_{k} P(k) p(k) x(k)$. Taking the derivative with respect to $p(s)$ we have

$$
\frac{\partial \Pi_{\text {out }}}{\partial p(s)}=P(s)\left[1-2 p(s)+\frac{\gamma(1-\bar{p})}{1-\gamma \bar{k}} s\right]-\sum_{k} P(k) p(k) \frac{\gamma k}{1-\gamma \bar{k}} \frac{\partial \bar{p}}{\partial p(s)} .
$$

Note that

$$
\frac{\partial \bar{p}}{\partial p(s)}=\sum_{l} \bar{H}(l) P(s \mid l)=P(s) \sum_{l} \frac{l H(l \mid s)}{\hat{k}}
$$

where the second equality follows because by Bayes' rule $P(s \mid l)=\frac{H(l \mid s) P(s)}{H(l)}$. Hence

$$
\frac{\partial \Pi_{\text {out }}}{\partial p(s)}=P(s)\left[1-2 p(s)+\frac{\gamma(1-\bar{p})}{1-\gamma \bar{k}} s-\frac{\gamma}{1-\gamma \bar{k}} \sum_{k} P(k) p(k) k \sum_{l} \frac{l H(l \mid s)}{\hat{k}}\right],
$$

and so the first order condition is

$$
1-2 p^{*}(s)+\frac{\gamma}{1-\gamma \bar{k}}\left[\left(1-\bar{p}^{*}\right) s-\sum_{k} P(k) p^{*}(k) k \sum_{l} \frac{l H(l \mid s)}{\hat{k}}\right]=0 .
$$

Rearranging and denoting $\phi=\sum_{k} P(k) p^{*}(k) k$ and $C(s)=\sum_{l} \frac{l H(l \mid s)}{\hat{k}}$, we have

$$
p^{*}(s)=\frac{1}{2}\left[1+\frac{\gamma}{1-\gamma \bar{k}}\left(\left(1-\bar{p}^{*}\right) s-\phi C(s)\right)\right] .
$$

Using the expression of $\bar{p}$ and of $p^{*}(s)$, and developing, we obtain

$$
\bar{p}^{*}=\frac{1}{2}\left[1+\frac{\gamma}{1-\gamma \bar{k}}\left[\left(1-\bar{p}^{*}\right) \bar{k}-\phi \frac{\sigma_{\hat{l}(k)}^{2}+\hat{k}^{2}}{\hat{k}^{2}}\right]\right] .
$$

Combining $\phi=\sum_{k} P(k) p^{*}(k) k$ and expression 7 , we derive:

$$
\phi=\frac{1}{2}\left[\hat{k}+\frac{\gamma}{1-\gamma \bar{k}}\left(\left(1-\bar{p}^{*}\right)\left(\sigma_{k}^{2}+\hat{k}^{2}\right)-\phi \bar{k}\right)\right] .
$$

Solving these two last equations for $\bar{p}^{*}$ and $\phi$, and then plugging these expressions back in expression 7 for $p^{*}(s)$, we obtain

$$
p^{*}(k)=\frac{1}{2}-\frac{\gamma \hat{k}}{2} \frac{\hat{k}[2-\gamma(\bar{k}-\hat{k})][\hat{l}(k)-k]+\gamma\left[\hat{l}(k) \sigma_{k}^{2}-k \sigma_{\hat{l}(k)}^{2}\right]}{\hat{k}^{2}(2-\bar{k} \gamma)^{2}-\gamma^{2}\left(\sigma_{\hat{l}(k)}^{2}+\hat{k}^{2}\right)\left(\sigma_{k}^{2}+\hat{k}^{2}\right)} .
$$


The demand of consumer with out-degree $k$ is $x(k)=1-p(k)+\frac{\gamma(1-\bar{p})}{1-\gamma \bar{k}} k$, and using expression 8 we obtain

$$
x^{*}(k)=\frac{1}{2}+\frac{\gamma \hat{k}}{2} \frac{\hat{k}[2-\gamma(\bar{k}-\hat{k})][\hat{l}(k)+k]+\gamma\left[\hat{l}(k) \sigma_{k}^{2}+k \sigma_{\hat{l}(k)}^{2}\right]}{\hat{k}^{2}(2-\bar{k} \gamma)^{2}-\gamma^{2}\left(\sigma_{\hat{l}(k)}^{2}+\hat{k}^{2}\right)\left(\sigma_{k}^{2}+\hat{k}^{2}\right)} .
$$

Finally, profits are

$$
\Pi_{\text {out }}^{*}=\sum_{k} P(k) \sum_{l} H(l \mid k) p^{*}(k) x^{*}(k)=\sum_{k} P(k) p^{*}(k) x^{*}(k) .
$$

Discrimination on the in-degree. We have $p(k, l)=p(l)$ and

$$
x(k, l)=1-p(l)+\frac{\gamma(1-\bar{p})}{1-\gamma \bar{k}} k
$$

where $\bar{p}=\frac{1}{\hat{k}} \sum_{l} H(l) l p(l)$. The profits are $\Pi_{i n}=\sum_{l} H(l) \sum_{k} P(k \mid l) p(l) x(k, l)$, and

$$
\frac{\partial \Pi_{i n}}{\partial p(s)}=H(s) \sum_{k} P(k \mid s)\left[1-2 p(s)+\frac{\gamma(1-\bar{p})}{1-\gamma \bar{k}} k\right]-\sum_{l} H(l) \sum_{k} P(k \mid l) \frac{p(l) k \gamma}{1-\gamma \bar{k}} \frac{\partial \bar{p}}{\partial p(s)}
$$

Using the fact that $\frac{\partial \bar{p}}{\partial p(s)}=\frac{1}{\hat{k}} H(s) s$ and that $\hat{k}(l)=\sum_{k} P(k \mid l) k$, we can rewrite the first order condition as

$$
H(s)\left[1-2 p^{*}(s)+\frac{\gamma}{1-\gamma \bar{k}}\left(\left(1-\bar{p}^{*}\right) \hat{k}(s)-\frac{s}{\hat{k}} \sum_{l} H(l) p^{*}(l) \hat{k}(l)\right)\right]=0,
$$

which holds if and only if

$$
p^{*}(s)=\frac{1}{2}\left[1+\frac{\gamma}{1-\gamma \bar{k}}\left(\left(1-\bar{p}^{*}\right) \hat{k}(s)-\frac{s}{\hat{k}} \sum_{l} H(l) p^{*}(l) \hat{k}(l)\right)\right] .
$$

Defining $\phi=\frac{1}{\hat{k}} \sum_{l} H(l) p^{*}(l) \hat{k}(l)$, we have that

$$
p^{*}(s)=\frac{1}{2}\left[1+\frac{\gamma}{1-\gamma \bar{k}}\left(\left(1-\bar{p}^{*}\right) \hat{k}(s)-s \phi\right)\right]
$$

Using $p^{*}(s)$ and the definition of $\bar{p}$ we obtain that

$$
\bar{p}^{*}=\frac{1}{\hat{k}} \sum_{l} H(l) l p^{*}(l)=\frac{1}{2}\left[1+\frac{\gamma}{1-\gamma \bar{k}}\left(\left(1-\bar{p}^{*}\right) \bar{k}-\phi \frac{\sigma_{l}^{2}+\hat{k}^{2}}{\hat{k}}\right)\right] .
$$

Similarly, using $p^{*}(s)$ and the definition of $\phi$ we obtain

$$
\phi=\frac{1}{\hat{k}} \sum_{l} H(l) p^{*}(l) \hat{k}(l)=\frac{1}{2}\left[1+\frac{\gamma}{1-\gamma \bar{k}}\left(\left(1-\bar{p}^{*}\right) \frac{\sigma_{\hat{k}(l)}^{2}+\hat{k}^{2}}{\hat{k}}-\phi \bar{k}\right)\right] .
$$


Solving the two equations for $\phi$ and for $\bar{p}^{*}$, and plugging the obtained expressions on the expression for $p^{*}(l)$, we have

$$
p^{*}(l)=\frac{1}{2}+\frac{\gamma \hat{k}}{2} \frac{\hat{k}[2-\gamma(\bar{k}-\hat{k})][\hat{k}(l)-l]+\gamma\left[\hat{k}(l) \sigma_{l}^{2}-l \sigma_{\hat{k}(l)}^{2}\right]}{\hat{k}^{2}(2-\bar{k} \gamma)^{2}-\gamma^{2}\left(\sigma_{\hat{k}(l)}^{2}+\hat{k}^{2}\right)\left(\sigma_{l}^{2}+\hat{k}^{2}\right)} .
$$

Next, note that $x(k, l)=1-p(l)+\frac{\gamma(1-\bar{p})}{1-\gamma \bar{k}} k$, and using the expression for $p^{*}(l)$ we obtain

$$
x^{*}(k, l)=\frac{1}{2}+\frac{\gamma \hat{k}}{2} \frac{\hat{k}[2-\gamma(\bar{k}-\hat{k})][l-\hat{k}(l)+2 k]+\gamma\left[l \sigma_{\hat{k}(l)}^{2}-\hat{k}(l) \sigma_{l}^{2}+2 k \sigma_{l}^{2}\right]}{\hat{k}^{2}(2-\bar{k} \gamma)^{2}-\gamma^{2}\left(\sigma_{\hat{k}(l)}^{2}+\hat{k}^{2}\right)\left(\sigma_{l}^{2}+\hat{k}^{2}\right)} .
$$

We can finally derive an expression of the profit by noticing that

$$
\Pi_{i n}^{*}=\sum_{l} H(l) p^{*}(l) \sum_{k} P(k \mid l) x^{*}(k, l) .
$$

Discrimination on in-degree and out-degree. We employ similar steps of derivation.

$$
x(k, l)=1-p(k, l)+\frac{\gamma(1-\bar{p})}{1-\gamma \bar{k}} k
$$

where $\bar{p}=\sum_{l} \bar{H}(l) \sum_{k} P(k \mid l) p(k, l)$. The profits are $\Pi_{\text {in } / \text { out }}=\sum_{l} H(l) \sum_{k} P(k \mid l) p(k, l) x(k, l)$, and

$$
\frac{\partial \Pi_{\text {in } / \text { out }}}{\partial p(x, y)}=H(y) P(x \mid y)\left[1-2 p(x, y)+\frac{\gamma(1-\bar{p})}{1-\gamma \bar{k}} x\right]-\sum_{l} H(l) \sum_{k} P(k \mid l) \frac{p(k, l) k \gamma}{1-\gamma \bar{k}} \frac{\partial \bar{p}}{\partial p(x, y)}
$$

and using the fact that $\frac{\partial \bar{p}}{\partial p(x, y)}=\frac{H(y) P(x \mid y) y}{\hat{k}}$ we can rewrite the first order condition as

$$
p^{*}(x, y)=\frac{1}{2}\left[1+\frac{\gamma}{1-\gamma \bar{k}}\left(\left(1-\bar{p}^{*}\right) x-\phi y\right)\right]
$$

where $\phi=\frac{1}{\hat{k}} \sum_{l} H(l) \sum_{k} P(k \mid l) k p^{*}(k, l)$. Using $p(x, y)$ and the definition of $\bar{p}$ we obtain that

$$
\bar{p}^{*}=\frac{1}{2}\left[1+\frac{\gamma}{1-\gamma \bar{k}}\left(\left(1-\bar{p}^{*}\right) \bar{k}-\phi \frac{\sigma_{l}^{2}+\hat{k}^{2}}{\hat{k}}\right)\right] .
$$

Similarly, using $p^{*}(x, y)$ and the definition of $\phi$ we obtain

$$
\phi=\frac{1}{2}\left[1+\frac{\gamma}{1-\gamma \bar{k}}\left(\left(1-\bar{p}^{*}\right) \frac{\sigma_{k}^{2}+\hat{k}^{2}}{\hat{k}}-\phi \bar{k}\right)\right] .
$$

Solving the two equations for $\phi$ and for $\bar{p}^{*}$, and plugging these two expressions in the expression 
for $p^{*}(k, l)$, we obtain

$$
p^{*}(k, l)=\frac{1}{2}+\frac{1}{2} \frac{\gamma \hat{k}^{2}[\gamma(\bar{k}-\hat{k})-2][l-k]+\gamma^{2} \hat{k}\left[k \sigma_{l}^{2}-l \sigma_{k}^{2}\right]}{\hat{k}^{2}(2-\bar{k} \gamma)^{2}-\gamma^{2}\left(\sigma_{k}^{2}+\hat{k}^{2}\right)\left(\sigma_{l}^{2}+\hat{k}^{2}\right)} .
$$

Next, recall that

$$
x^{*}(k, l)=1-p^{*}(k, l)+\frac{\gamma\left(1-\bar{p}^{*}\right)}{1-\gamma \bar{k}} k
$$

and that profit is given by

$$
\Pi_{\text {in } / \text { out }}^{*}=\sum_{k} \sum_{l} H(l \mid k) p^{*}(k, l) x^{*}(k, l) .
$$

Proposition 11. Suppose that $\gamma k^{\max }<\frac{1}{2}$. Then, for any of the price discrimination schemes considered, there exists a unique finite price schedule $\mathbf{p}$ that solves the monopoly's profit maximization problem.

The proof of Proposition 11 is available in an online appendix on the authors' webpages.

\section{Appendix B.}

Proof of proposition 1. Independence between in-degree and out-degree implies that $\bar{k}=\hat{k}$. Thus, the proposition follows directly from Proposition 10 which proves the same result for the general setup with any correlation level between in- and out-degrees.

Proof of proposition 2. Independence between in-degree and out-degree implies that $\bar{k}=\hat{k}$ and so the consumption of a consumer with out-degree $k$ and in-degree $l$ equals

$$
x(k, l)=x(k)=\frac{1}{2}\left(1+\frac{\gamma k}{1-\gamma \hat{k}}\right) .
$$

and the expected monopoly equilibrium profit is

$$
\Pi=\frac{1}{4} \cdot \frac{1}{1-\gamma \hat{k}}
$$

The comparative statics are immediate.

Proof of Proposition 3. Independence between in-degree and out-degree implies that $\bar{k}=\hat{k}$, $\hat{k}(l)=\hat{k}$ for all in-degree $l, \hat{l}(k)=\hat{k}$ for all in-degree $l, \sigma_{\hat{l}(k)}^{2}=0$, and $\sigma_{\hat{k}(l)}^{2}=0$. Imposing these conditions in expressions 8,13 , and 17 separately and rearranging, we obtain the optimal price scheme for the case of price discrimination on out-degrees, in-degrees and both, respectively:

$$
\begin{gathered}
p(k)=\frac{1}{2}-\frac{\gamma}{2}\left[\frac{\gamma \sigma_{k}^{2}+\hat{k}-k}{4-4 \gamma \hat{k}-\gamma^{2} \sigma_{k}^{2}}\right], \\
p(l)=\frac{1}{2}+\frac{\gamma}{2}\left[\frac{\gamma \sigma_{l}^{2}+\hat{k}-l}{4-4 \gamma \hat{k}-\gamma^{2} \sigma_{l}^{2}}\right],
\end{gathered}
$$


and

$$
p(k, l)=\frac{1}{2}+\frac{\gamma \hat{k}}{2}\left[\frac{\left(\gamma \sigma_{l}^{2}+2 \hat{k}\right) k-\left(\gamma \sigma_{k}^{2}+2 \hat{k}\right) l}{\hat{k}^{2}\left[4-4 \gamma \hat{k}-\gamma^{2}\left(\sigma_{k}^{2}+\sigma_{l}^{2}\right)\right]-\gamma^{2} \sigma_{k}^{2} \sigma_{l}^{2}}\right]
$$

It is straightforward to verify the remaining part of the proposition.

Proof of Proposition 4. Independence between in-degree and out-degree implies that $\bar{k}=\hat{k}$, $\hat{k}(l)=\hat{k}$ for all in-degree $l, \hat{l}(k)=\hat{k}$ for all in-degree $l, \sigma_{\hat{l}(k)}^{2}=0$, and $\sigma_{\hat{k}(l)}^{2}=0$. Imposing these conditions in expressions 9,14 , and 18 separately and rearranging, we obtain equilibrium consumption for a consumer with out-degree $k$ and in-degree $l$ when the monopoly discriminate on out-degrees, in-degrees and both, respectively:

$$
\begin{gathered}
x(k, l)=x(k)=\frac{2+\gamma(k-\hat{k})}{4-4 \gamma \hat{k}-\gamma^{2} \sigma_{k}^{2}} \\
x(k, l)=1-\frac{2-\gamma \hat{k}-\gamma l}{4-4 \gamma \hat{k}-\gamma^{2} \sigma_{l}^{2}}+\frac{\gamma\left(2 \hat{k}+\gamma \sigma_{l}^{2}\right)}{\hat{k}\left(4-4 \gamma \hat{k}-\gamma^{2} \sigma_{l}^{2}\right)} k
\end{gathered}
$$

and

$$
x(k, l)=\frac{1}{2}\left[1+\frac{\hat{k} \gamma}{\hat{k}^{2}\left[4-4 \hat{k} \gamma-\gamma^{2}\left(\sigma_{k}^{2}+\sigma_{l}^{2}\right)\right]-\gamma^{2} \sigma_{l}^{2} \sigma_{k}^{2}}\left(\left(\gamma \sigma_{l}^{2}+2 \hat{k}\right) k+\left(\gamma \sigma_{k}^{2}+2 \hat{k}\right) l\right)\right]
$$

It is straightforward to verify the remaining part of the proposition.

\section{Proof of Proposition 5}

Discrimination on out-degree. Recall that the price is given by expression 21 and the demand by expression 24 . Using $p(k)$ and $x(k)$ we can derive the optimal profit:

$$
\Pi_{\text {out }}=\frac{1}{4-4 \gamma \hat{k}-\gamma^{2} \sigma_{k}^{2}}
$$

Note that $4-4 \gamma \hat{k}-\gamma^{2} \sigma_{k}^{2}>0$, because $4-4 \gamma \hat{k}-\gamma^{2} \sigma_{k}^{2}>4-2-\gamma^{2}\left(k^{\max }\right)^{2}+\gamma^{2} \hat{k}^{2}>2-\frac{1}{4}+\gamma^{2} \hat{k}^{2}>$ 0 . The comparative statics are immediate.

Discrimination on in-degree. Recall that the price is given by expression 22 and the demand by expression 25 . Using $p(k)$ and $x(k)$ we can derive the optimal profit:

$$
\Pi_{i n}=\frac{1}{4-4 \gamma \hat{k}-\gamma^{2} \sigma_{l}^{2}}
$$

The comparative statics are immediate.

Discrimination on out-degree and in-degree. Recall that the price is given by expression 23 and the demand by expression 26 . Using $p(k)$ and $x(k)$ we can derive the optimal profit:

$$
\Pi_{\text {in } / \text { out }}=\frac{1}{4} \frac{4 \hat{k}^{2}-\gamma^{2} \sigma_{k}^{2} \sigma_{l}^{2}}{4 \hat{k}^{2}(1-\gamma \hat{k})-\gamma^{2} \hat{k}^{2}\left(\sigma_{k}^{2}+\sigma_{l}^{2}\right)-\gamma^{2} \sigma_{l}^{2} \sigma_{k}^{2}} .
$$


Note that $4 \hat{k}^{2}(1-\gamma \hat{k})-\gamma^{2} \hat{k}^{2}\left(\sigma_{k}^{2}+\sigma_{l}^{2}\right)-\gamma^{2} \sigma_{l}^{2} \sigma_{k}^{2}>0$, because $4 \hat{k}^{2}(1-\gamma \hat{k})-\gamma^{2} \hat{k}^{2}\left(\sigma_{k}^{2}+\right.$ $\left.\sigma_{l}^{2}\right)-\gamma^{2} \sigma_{l}^{2} \sigma_{k}^{2}>2 \hat{k}^{2}-\gamma^{2} \hat{k}^{2} \sigma_{k}^{2}-\gamma^{2} \hat{k}^{2} \sigma_{l}^{2}-\gamma^{2} \sigma_{l}^{2} \sigma_{k}^{2}>\hat{k}^{2}-\gamma^{2} \sigma_{l}^{2} \sigma_{k}^{2}$, sign $\left\{\hat{k}^{2}-\gamma^{2} \sigma_{l}^{2} \sigma_{k}^{2}\right\}=$ $\operatorname{sign}\left\{\hat{k}-\gamma \sigma_{l} \sigma_{k}\right\}$, and $\hat{k}-\gamma \sigma_{l} \sigma_{k}=\frac{1}{k^{\max }}\left(\hat{k} k^{\max }-\gamma k^{\max } \sigma_{l} \sigma_{k}\right)>\frac{1}{k^{\max }}\left(\hat{k} k^{\max }-\sigma_{l} \sigma_{k}\right)>$ $\frac{1}{k^{\max }}\left(\hat{k} k^{\max }-\max \left(\sigma_{l}^{2}, \sigma_{k}^{2}\right)\right)>$

$>\frac{1}{k^{\max }}\left(\hat{k} k^{\max }-\max \left(\sum_{l} H(l) l^{2}, \sum_{k} P(k) k^{2}\right)\right)=$

$=\frac{1}{k^{\max }}\left(\hat{k} k^{\max }-\max \left(\sum_{l} H(l) l\left(k^{\max }-l\right), \sum_{k} P(k) k\left(k^{\max }-k\right)\right)\right)>0$.

It is straightforward to verify that the comparative statics hold.

Proof of Corollary 2. Note that

$$
\Pi_{\text {out }}-\Pi_{\text {uniform }}=\frac{1}{4} \frac{\gamma^{2} \sigma_{k}^{2}}{\left(4-4 \gamma \hat{k}-\gamma^{2} \sigma_{k}^{2}\right)(1-\gamma \hat{k})},
$$

is increasing in $\sigma_{k}^{2}$ and $\hat{k}$. Similarly

$$
\Pi_{\text {in }}-\Pi_{\text {uniform }}=\frac{1}{4} \frac{\gamma^{2} \sigma_{x}^{2}}{\left(4-4 \gamma \hat{k}-\gamma^{2} \sigma_{x}^{2}\right)(1-\gamma \hat{k})}
$$

is increasing in $\sigma_{l}^{2}$ and $\hat{k}$. Comparing we obtain that the monopoly prefers to learn the outdegree than the in-degree if and only if $\sigma_{k}^{2}>\sigma_{l}^{2}$. Next, the value of the monopoly for learning both the in-degree and the out-degree relative of no-information is

$$
\Pi_{\text {in } / \text { out }}-\Pi_{\text {uniform }}=\frac{1}{4} \frac{\gamma^{2} \hat{k}\left[\hat{k}\left(\sigma_{l}^{2}+\sigma_{k}^{2}\right)+\gamma \sigma_{l}^{2} \sigma_{k}^{2}\right]}{(1-\gamma \hat{k})\left[\hat{k}^{2}\left(4-4 \hat{k} \gamma-\gamma^{2}\left(\sigma_{k}^{2}+\sigma_{l}^{2}\right)\right)-\gamma^{2} \sigma_{l}^{2} \sigma_{k}^{2}\right]},
$$

which is increasing in the variance of the in-degree and the variance of the out-degree.

Proof of Corollary 3. We say that the value for the monopoly from learning the out-degrees and in-degrees of all consumers exhibits complementarity if

$$
\Pi_{\text {in } / \text { out }}-\Pi_{\text {out }} \geq \Pi_{\text {in }}-\Pi_{\text {uniform }}
$$

We can re-write 27 as follows

$$
\frac{1}{4} \frac{4 \hat{k}^{2}-\gamma^{2} \sigma_{k}^{2} \sigma_{l}^{2}}{\hat{k}^{2}\left[4-4 \gamma \hat{k}-\gamma^{2}\left(\sigma_{k}^{2}+\sigma_{l}^{2}\right)\right]-\gamma^{2} \sigma_{k}^{2} \sigma_{l}^{2}}-\frac{1}{4-4 \gamma \hat{k}-\gamma^{2} \sigma_{k}^{2}} \geq \frac{1}{4-4 \gamma \hat{k}-\gamma^{2} \sigma_{l}^{2}}-\frac{1}{4} \cdot \frac{1}{1-\gamma \hat{k}}
$$

or equivalently

$$
\hat{k}\left(8-8 \hat{k} \gamma-3 \sigma_{k}^{2} \gamma^{2}-3 \sigma_{l}^{2} \gamma^{2}\right)+\gamma \sigma_{k}^{2}\left(2-\sigma_{l}^{2} \gamma^{2}\right)+2 \sigma_{l}^{2} \gamma \geq 0
$$

which holds for the entire range of parameters.

Proof of Proposition 6. Part 1: Consider a consumer with out-degree $k$ (and any indegree). The additional consumption of the consumer from the move from no discrimination to discrimination of out-degree is 


$$
\frac{2+\gamma(k-\hat{k})}{4-4 \gamma \hat{k}-\gamma^{2} \sigma_{k}^{2}}-\frac{1-\gamma \hat{k}+\gamma k}{2-2 \gamma \hat{k}}
$$

Some rearranging leads to the following equality

$$
\operatorname{sign}\left\{\frac{2+\gamma(k-\hat{k})}{4-4 \gamma \hat{k}-\gamma^{2} \sigma_{k}^{2}}-\frac{1-\gamma \hat{k}+\gamma k}{2-2 \gamma \hat{k}}\right\}=\operatorname{sign}\left\{-2 \hat{k}^{2} \gamma-\hat{k} \sigma_{k}^{2} \gamma^{2}+2 k \hat{k} \gamma+2 \hat{k}+k \sigma_{k}^{2} \gamma^{2}+\sigma_{k}^{2} \gamma-2 k\right\} .
$$

Finally, noting that

$$
\frac{\partial}{\partial k}\left(-2 \hat{k}^{2} \gamma-\hat{k} \sigma_{k}^{2} \gamma^{2}+2 k \hat{k} \gamma+2 \hat{k}+k \sigma_{k}^{2} \gamma^{2}+\sigma_{k}^{2} \gamma-2 k\right)=2 \hat{k} \gamma+\sigma_{k}^{2} \gamma^{2}-2<0,
$$

and evaluate $\operatorname{sign}\left\{-2 \hat{k}^{2} \gamma-\hat{k} \sigma_{k}^{2} \gamma^{2}+2 k \hat{k} \gamma+2 \hat{k}+k \sigma_{k}^{2} \gamma^{2}+\sigma_{k}^{2} \gamma-2 k\right\}$ for $k=\hat{k}$ and for $k=$ $k^{\max }$ complete the proof of part 1 .

Part 2: Consider a consumer with out-degree $k$ and in-degree $l$. The additional consumption of the consumer from the move from no discrimination to discrimination of in-degree is

$$
1-\frac{2-\gamma \hat{k}-\gamma l}{4-4 \gamma \hat{k}-\gamma^{2} \sigma_{l}^{2}}+\frac{\gamma\left(2 \hat{k}+\gamma \sigma_{l}^{2}\right)}{\hat{k}\left(4-4 \gamma \hat{k}-\gamma^{2} \sigma_{l}^{2}\right)} k-\frac{1}{2}\left(1+\frac{\gamma k}{1-\gamma \hat{k}}\right) .
$$

Some rearranging leads to the following equality

$$
\begin{aligned}
& \operatorname{sign}\left\{1-\frac{2-\gamma \hat{k}-\gamma l}{4-4 \gamma \hat{k}-\gamma^{2} \sigma_{l}^{2}}+\frac{\gamma\left(2 \hat{k}+\gamma \sigma_{l}^{2}\right)}{\hat{k}\left(4-4 \gamma \hat{k}-\gamma^{2} \sigma_{l}^{2}\right)} k-\frac{1}{2}\left(1+\frac{\gamma k}{1-\gamma \hat{k}}\right)\right\} \\
= & \operatorname{sign}\left\{2 \hat{k}^{3} \gamma+\hat{k}^{2} \sigma_{l}^{2} \gamma^{2}-2 l \hat{k}^{2} \gamma-2 \hat{k}^{2}-k \hat{k} \sigma_{l}^{2} \gamma^{2}-\hat{k} \sigma_{l}^{2} \gamma+2 l \hat{k}+2 k \sigma_{l}^{2} \gamma\right\} \equiv L .
\end{aligned}
$$

The proof of part 2 then follows by noticing that $\frac{\partial L}{\partial k}=\sigma_{l}^{2} \gamma(2-\gamma \hat{k})>0$ and $\frac{\partial L}{\partial l}=2 \hat{k}(1-\hat{k} \gamma)>$ 0 .

Proof of Proposition 7. The additional aggregate consumer surplus from discriminating on in-degree relative to no discrimination is

$$
\begin{aligned}
\Delta C S & =\frac{1}{2} \sum H(l) P(k)\left(\left(1-\frac{2-\gamma \hat{k}-\gamma l}{4-4 \gamma \hat{k}-\gamma^{2} \sigma_{l}^{2}}+\frac{\gamma\left(2 \hat{k}+\gamma \sigma_{l}^{2}\right)}{\hat{k}\left(4-4 \gamma \hat{k}-\gamma^{2} \sigma_{l}^{2}\right)} k\right)^{2}-\left(\frac{1}{2}\left(1+\frac{\gamma k}{1-\gamma \hat{k}}\right)\right)^{2}\right) \\
& =\frac{\gamma^{2} \sigma_{l}^{2}}{8 \hat{k}^{2}} \frac{4 \hat{k}^{4} \gamma^{2}+8 \hat{k}^{3} \sigma_{k}^{2} \gamma^{3}-16 \hat{k}^{3} \gamma+3 \hat{k}^{2} \sigma_{l}^{2} \sigma_{k}^{2} \gamma^{4}-\hat{k}^{2} \sigma_{l}^{2} \gamma^{2}-24 \hat{k}^{2} \sigma_{k}^{2} \gamma^{2}+12 \hat{k}^{2}-8 \hat{k} \sigma_{l}^{2} \sigma_{k}^{2} \gamma^{3}}{(1-\hat{k} \gamma)^{2}\left(4-4 \hat{k} \gamma-\sigma_{l}^{2} \gamma^{2}\right)^{2}} \\
& +\frac{\gamma^{2} \sigma_{l}^{2}}{8 \hat{k}^{2}} \frac{16 \hat{k} \sigma_{k}^{2} \gamma+4 \sigma_{l}^{2} \sigma_{k}^{2} \gamma^{2}}{(1-\hat{k} \gamma)^{2}\left(4-4 \hat{k} \gamma-\sigma_{l}^{2} \gamma^{2}\right)^{2}}
\end{aligned}
$$


Which has the same sign as

$$
\begin{aligned}
& 4 \hat{k}^{4} \gamma^{2}+8 \hat{k}^{3} \sigma_{k}^{2} \gamma^{3}-16 \hat{k}^{3} \gamma+3 \hat{k}^{2} \sigma_{l}^{2} \sigma_{k}^{2} \gamma^{4}-\hat{k}^{2} \sigma_{l}^{2} \gamma^{2}-24 \hat{k}^{2} \sigma_{k}^{2} \gamma^{2}+12 \hat{k}^{2}-8 \hat{k} \sigma_{l}^{2} \sigma_{k}^{2} \gamma^{3}+16 \hat{k} \sigma_{k}^{2} \gamma+4 \sigma_{l}^{2} \sigma_{k}^{2} \gamma^{2} \\
= & 4 \hat{k}^{4} \gamma^{2}-16 \hat{k}^{3} \gamma-\hat{k}^{2} \sigma_{l}^{2} \gamma^{2}+12 \hat{k}^{2}+\gamma \sigma_{k}^{2}\left(16 \hat{k}-24 \hat{k}^{2} \gamma-8 \hat{k} \sigma_{l}^{2} \gamma^{2}+8 \hat{k}^{3} \gamma^{2}+3 \hat{k}^{2} \sigma_{l}^{2} \gamma^{3}+4 \sigma_{l}^{2} \gamma\right)>0 .
\end{aligned}
$$

\section{Proof of Proposition 8.}

Uniform price. Using $\rho=\frac{\bar{k}-\hat{k}}{\sigma^{2}} \hat{k}$ we can rewrite the expression for $\Pi_{\text {uniform }}^{*}$ to obtain

$$
\Pi_{\text {uniform }}^{*}=\frac{\hat{k}-\gamma \rho \sigma^{2}}{4\left(\hat{k}-\gamma \rho \sigma^{2}\right)-4 \gamma \hat{k}^{2}} .
$$

Discrimination out-degree. We first consider the case of price discrimination in out-degree. Assumption 1 and $\sigma_{l}^{2}=\sigma_{k}^{2}$ imply that $\hat{l}(k)=\hat{k}+\frac{\hat{k}(\bar{k}-\hat{k})(k-\hat{k})}{\sigma_{k}^{2}}$ and $\sigma_{\hat{l}(k)}^{2}=\rho^{2} \sigma_{l}^{2}$. We can then rewrite the expression of the price charged to consumer with out-degree $k$ as

$$
p(k)=\frac{1}{2}+\frac{1}{2} \frac{(1-\rho) \gamma\left[2(k-\hat{k})\left(\hat{k}-\rho \gamma \sigma^{2}\right)-\gamma \sigma^{2} \hat{k}(1+\rho)\right]}{4\left[\hat{k}-\gamma \rho \sigma^{2}\right]-4 \gamma \hat{k}^{2}-\gamma^{2} \sigma^{2} \hat{k}(1-\rho)^{2}},
$$

and the consumption of an agent with out-degree $k$ is

$$
x(k)=\frac{1}{2}+\frac{1}{2} \frac{\hat{k}\left(\gamma^{2} \sigma^{2}-\gamma^{2} \rho \sigma^{2}+2 \gamma \hat{k}\right)(1-\rho)+2 \gamma \hat{k}(1+\rho) k}{4\left(\hat{k}-\gamma \rho \sigma^{2}\right)-4 \gamma \hat{k}^{2}-\gamma^{2} \sigma^{2} \hat{k}(1-\rho)^{2}},
$$

and computing profits we obtain

$$
\Pi_{\text {out }}^{*}=\frac{\hat{k}-\gamma \rho \sigma^{2}}{4\left(\hat{k}-\gamma \rho \sigma^{2}\right)-4 \gamma \hat{k}^{2}-\gamma^{2} \sigma^{2} \hat{k}(1-\rho)^{2}} .
$$

Discrimination in-degree. We now consider the case of price discrimination in the indegree. In this case, under assumption 1 and $\sigma_{l}^{2}=\sigma_{k}^{2}=\sigma^{2}$ we can specialize the expression for the price charged to a consumer with in-degree $l$ as follows

$$
p(l)=\frac{1}{2}-\frac{1}{2} \frac{(1-\rho) \gamma\left[2(l-\hat{k})\left(\hat{k}-\rho \gamma \sigma^{2}\right)-\gamma \sigma^{2} \hat{k}(1+\rho)\right]}{4\left[\hat{k}-\gamma \rho \sigma^{2}\right]-4 \gamma \hat{k}^{2}-\gamma^{2} \sigma^{2} \hat{k}(1-\rho)^{2}}
$$

and the consumption of a consumer with in-degree $l$ and out-degree $k$ is

$$
x(l, k)=\frac{1}{2}+\frac{1}{2} \frac{\gamma \hat{k}\left[((l-\hat{k})(1-\rho)+2 k)\left(2 \hat{k}-\rho \sigma^{2} \gamma\right)+\gamma\left(l \rho^{2} \sigma^{2}-\sigma^{2}(\hat{k}+\rho(l-\hat{k}))+2 k \sigma^{2}\right)\right]}{\left[2 \hat{k}-\gamma\left(\rho \sigma^{2}+\hat{k}^{2}\right)\right]^{2}-\gamma^{2}\left(\sigma^{2}+\hat{k}^{2}\right)\left(\rho \sigma^{2}+\hat{k}^{2}\right)} .
$$

Computing profits we obtain the same expression in the case of discrimination on out-degree. Hence, the expected profits under discrimination in in-degree or in out-degree are the same, given assumption 1 and the fact that $\sigma_{l}^{2}=\sigma_{k}^{2}$. 
We now show that $\Pi_{\text {out }}^{*}$ and $\Pi_{\text {in }}^{*}$ are increasing in $\rho$. First note that we can rewrite $\Pi_{\text {out }}^{*}$ as

$$
\Pi_{\text {out }}^{*}=\frac{1}{4}+\frac{1}{4} \frac{\gamma^{2} \sigma^{2} \hat{k}(1-\rho)^{2}+4 \gamma \hat{k}^{2}}{4\left(\hat{k}-\gamma \rho \sigma^{2}\right)-4 \gamma \hat{k}^{2}-\gamma^{2} \sigma^{2} \hat{k}(1-\rho)^{2}}
$$

and that

$$
\begin{aligned}
\operatorname{sign}\left\{\frac{\partial \Pi_{\text {out }}^{*}}{\partial \rho}\right\} & =\operatorname{sign}\left\{\frac{\partial}{\partial \rho}\left(\frac{\gamma^{2} \sigma^{2} \hat{k}(1-\rho)^{2}+4 \gamma \hat{k}^{2}}{4\left(\hat{k}-\gamma \rho \sigma^{2}\right)-4 \gamma \hat{k}^{2}-\gamma^{2} \sigma^{2} \hat{k}(1-\rho)^{2}}\right)\right\} \\
& =-\operatorname{sign}\left\{\frac{\partial}{\partial \rho}\left(\frac{\hat{k}-\gamma \rho \sigma^{2}}{\gamma^{2} \sigma^{2} \hat{k}(1-\rho)^{2}+4 \gamma \hat{k}^{2}}\right)\right\}
\end{aligned}
$$

and

$$
\begin{gathered}
\operatorname{sign}\left\{\frac{\partial}{\partial \rho}\left(\frac{\hat{k}-\gamma \rho \sigma^{2}}{\gamma^{2} \sigma^{2} \hat{k}(1-\rho)^{2}+4 \gamma \hat{k}^{2}}\right)\right\} \\
=\operatorname{sign}\left\{-\hat{k} \sigma^{2} \gamma^{2}(\rho+1)\left(2 \hat{k}+\sigma^{2} \gamma(1-\rho)\right)\right\} .
\end{gathered}
$$

Discrimination in/out-degree. We now turn to the case of price discrimination in both dimensions. First, by using the expression for the price charged to a consumer with out-degree $k$ and in-degree $l$ derived for general correlation structures and imposing assumption 1 and the assumption that $\sigma_{l}^{2}=\sigma_{k}^{2}$ we obtain that

$$
p(k, l)=\frac{1}{2}+\frac{1}{2} \frac{\gamma \hat{k}[k-l]\left[\gamma \sigma^{2}(1-\rho)+2 \hat{k}\right]}{\left[2 \hat{k}-\gamma\left(\rho \sigma^{2}+\hat{k}^{2}\right)\right]^{2}-\gamma^{2}\left(\sigma^{2}+\hat{k}^{2}\right)^{2}} .
$$

It is convenient to define

$$
A=\frac{\gamma \hat{k}\left[\gamma \sigma^{2}(1-\rho)+2 \hat{k}\right]}{\left[2 \hat{k}-\gamma\left(\rho \sigma^{2}+\hat{k}^{2}\right)\right]^{2}-\gamma^{2}\left(\sigma^{2}+\hat{k}^{2}\right)^{2}},
$$

so that $p(k, l)=\frac{1}{2}+\frac{1}{2} A[k-l]$. Using this expression we can calculate $\phi$ and $\bar{p}$ and obtain that

$$
\begin{aligned}
& \phi=\frac{1}{\hat{k}} \sum_{l} H(l) \sum_{k} P(k \mid l) k p(k, l)=\frac{1}{2}+\frac{1}{2 \hat{k}} A \sigma^{2}(1-\rho) \\
& \bar{p}=\frac{1}{\hat{k}} \sum_{l} H(l) \sum_{k} P(k \mid l) l p(k, l)=\frac{1}{2}-\frac{1}{2 \hat{k}} A \sigma^{2}(1-\rho) .
\end{aligned}
$$

Furthermore, we can develop an expression of

$$
\sum_{l} H(l) \sum_{k} P(k \mid l) p(k, l)^{2}=\frac{1}{4}+\frac{1}{2} A^{2}\left[\sigma^{2}(1-\rho)\right]^{2} .
$$


Since

$$
\Pi_{\text {in } / \text { out }}=\sum_{k} \sum_{l} H(l \mid k) p(k, l)^{2}+\frac{\gamma \phi \bar{p} \hat{k}}{1-\gamma \bar{k}},
$$

by substituting the above expression and simplifying we obtain

$$
\Pi_{\text {in } / \text { out }}^{*}=\frac{1}{4} \frac{2 \hat{k}-\gamma \sigma^{2}(1+\rho)}{2 \hat{k}(1-\gamma \hat{k})-\gamma \sigma^{2}(1+\rho)} .
$$

Taking the derivatives of the profit with respect to $\rho$ we obtain

$$
\frac{\partial \Pi_{\text {in } / \text { out }}}{\partial \rho}=\frac{1}{2} \frac{\gamma^{2} \hat{k}^{2} \sigma^{2}}{\left[2 \hat{k}(1-\gamma \hat{k})-\gamma \sigma^{2}(1+\rho)\right]^{2}}>0
$$

This concludes the proof of Proposition 8.

\section{Proof of Proposition 9.}

First Part. The additional profit that a monopoly gets for being able to discriminate on out-degree relative to charge a uniform price is

$$
\begin{aligned}
\Delta \Pi_{k, 0}(\rho) & =\frac{1}{4}+\frac{1}{4} \frac{\gamma^{2} \sigma^{2} \hat{k}(1-\rho)^{2}+4 \gamma \hat{k}^{2}}{4\left(\hat{k}-\gamma \rho \sigma^{2}\right)-4 \gamma \hat{k}^{2}-\gamma^{2} \sigma^{2} \hat{k}(1-\rho)^{2}}-\left(\frac{1}{4}\left[1+\frac{\gamma \hat{k}}{1-\gamma \bar{k}}\right]\right) \\
& =\frac{\gamma^{2} \sigma^{2} \hat{k}}{4}\left(\frac{(1-\rho)^{2}\left(\hat{k}-\gamma \rho \sigma^{2}\right)}{\left(4 \hat{k}-4 \gamma \rho \sigma^{2}-4 \gamma \hat{k}^{2}-\gamma^{2} \sigma^{2} \hat{k}(1-\rho)^{2}\right)\left(\hat{k}-\gamma \rho \sigma^{2}-\gamma \hat{k}^{2}\right)}\right) .
\end{aligned}
$$

We observe that $\Delta \Pi_{k, 0}(1)=0$ and for any $\rho<1, \Delta \Pi_{k, 0}(\rho) \geq 0$. We now show that $\Delta \Pi_{k, 0}$ is decreasing in $\rho$. To see this note that

$$
\begin{aligned}
\operatorname{sign}\left\{\frac{\partial \Delta \Pi_{k, 0}}{\partial \rho}\right\} & =-\operatorname{sign}\left\{\frac{\partial}{\partial \rho}\left(\frac{\left(4 \hat{k}-4 \gamma \rho \sigma^{2}-4 \gamma \hat{k}^{2}-\gamma^{2} \sigma^{2} \hat{k}(1-\rho)^{2}\right)\left(\hat{k}-\gamma \rho \sigma^{2}-\gamma \hat{k}^{2}\right)}{(1-\rho)^{2}\left(\hat{k}-\gamma \rho \sigma^{2}\right)}\right)\right\} \\
& =-\operatorname{sign}\left\{\frac{\partial}{\partial \rho}\left(4 \frac{\left(\hat{k}-\gamma \rho \sigma^{2}-\gamma \hat{k}^{2}\right)^{2}}{(1-\rho)^{2}\left(\hat{k}-\gamma \rho \sigma^{2}\right)}+\frac{\gamma^{3} \sigma^{2} \hat{k}^{3}}{\hat{k}-\gamma \rho \sigma^{2}}\right)\right\} .
\end{aligned}
$$

Next, $\frac{\partial}{\partial \rho}\left(\frac{\gamma^{3} \sigma^{2} \hat{k}^{3}}{\hat{k}-\gamma \rho \sigma^{2}}\right) \geq 0$, and

$$
\operatorname{sign}\left\{\frac{\partial}{\partial \rho}\left(\frac{\left(\hat{k}-\gamma \rho \sigma^{2}-\gamma \hat{k}^{2}\right)^{2}}{(1-\rho)^{2}\left(\hat{k}-\gamma \rho \sigma^{2}\right)}\right)\right\}=\operatorname{sign}\{\xi(\rho)\},
$$

where $\xi(\rho)=-2 \gamma \sigma^{2}(1-\rho)\left(\hat{k}-\gamma \rho \sigma^{2}\right)+2\left(\hat{k}-\gamma \rho \sigma^{2}\right)\left(\hat{k}-\gamma \rho \sigma^{2}-\gamma \hat{k}^{2}\right)+\gamma \sigma^{2}(1-\rho)\left(\hat{k}-\gamma \rho \sigma^{2}-\gamma \hat{k}^{2}\right)$. 
Since

$$
\frac{\partial \xi}{\partial \rho}=\sigma^{2} \gamma\left(3 \hat{k}^{2} \gamma-3 \hat{k}+\sigma^{2} \gamma+2 \sigma^{2} \gamma \rho\right) \leq 0
$$

and

$$
\xi(1)=2\left(\hat{k}-\gamma \sigma^{2}\right)\left(\hat{k}-\gamma \sigma^{2}-\gamma \hat{k}^{2}\right) \geq 0
$$

it follows that $\xi(\rho) \geq 0$. This proves the claim that $\Delta \Pi_{k, 0}$ is decreasing in $\rho$.

Second Part. We now prove the second part of Proposition 9. By definition, the value for the monopoly from learning out-degrees and in-degrees of all consumers exhibits complementarity iff $\Pi_{\text {in } / \text { out }}^{*}-\Pi_{\text {out }}^{*} \geq \Pi_{\text {in }}^{*}-\Pi_{\text {uniform }}^{*}$, i.e., iff

$$
\begin{aligned}
0 & \leq \frac{1}{4} \frac{2 \hat{k}-\gamma \sigma^{2}(1+\rho)}{2 \hat{k}(1-\gamma \hat{k})-\gamma \sigma^{2}(1+\rho)}-2 \frac{\hat{k}-\gamma \rho \sigma^{2}}{4\left(\hat{k}-\gamma \rho \sigma^{2}\right)-4 \gamma \hat{k}^{2}-\gamma^{2} \sigma^{2} \hat{k}(1-\rho)^{2}}+\frac{\hat{k}-\gamma \rho \sigma^{2}}{4\left(\hat{k}-\gamma \rho \sigma^{2}\right)-4 \gamma \hat{k}^{2}} \\
& =\frac{-\hat{k} \sigma^{2} \gamma^{2}(1-\rho) \eta(\rho)}{4\left(-\gamma \hat{k}^{2}-\gamma \rho \sigma^{2}+\hat{k}\right)\left(-2 \gamma \hat{k}^{2}-\gamma \rho \sigma^{2}-\gamma \sigma^{2}+2 \hat{k}\right)\left(-\gamma^{2} \hat{k} \rho^{2} \sigma^{2}+2 \gamma^{2} \hat{k} \rho \sigma^{2}-\gamma^{2} \hat{k} \sigma^{2}-4 \gamma \hat{k}^{2}-4 \gamma \rho \sigma^{2}+4 \hat{k}\right)}
\end{aligned}
$$

where

$\eta(\rho)=4 \hat{k}^{3} \gamma \rho-3 \hat{k}^{2} \sigma^{2} \gamma^{2} \rho^{2}+2 \hat{k}^{2} \sigma^{2} \gamma^{2} \rho+\hat{k}^{2} \sigma^{2} \gamma^{2}-4 \hat{k}^{2} \rho+6 \hat{k} \sigma^{2} \gamma \rho^{2}-2 \hat{k} \sigma^{2} \gamma-2 \sigma^{4} \gamma^{2} \rho^{3}+2 \sigma^{4} \gamma^{2} \rho$.

The following claim is sufficient to establish the desired result.

Claim 1. The following holds
a. $\hat{k}-\gamma \hat{k}^{2}-\gamma \rho \sigma^{2}>0$.
b. $2 \hat{k}-2 \gamma \hat{k}^{2}-\gamma \sigma^{2}-\gamma \rho \sigma^{2}>0$.
c. $4 \hat{k}-4 \gamma \hat{k}^{2}-\gamma^{2} \hat{k} \sigma^{2}-4 \gamma \rho \sigma^{2}+2 \gamma^{2} \hat{k} \rho \sigma^{2}-\gamma^{2} \hat{k} \rho^{2} \sigma^{2}>0$.
d. $\eta(\rho)$ changes from positive to negative at a unique $\rho<0$.

Proof of Claim 1. To see part a note that $\operatorname{sign}\left\{\hat{k}-\gamma \hat{k}^{2}-\gamma \rho \sigma^{2}\right\}=\operatorname{sign}\left\{1-\frac{1}{\hat{k}} \gamma \hat{k}^{2}-\frac{1}{\hat{k}} \gamma \rho \sigma^{2}\right\}>$ 0 , where the inequality follows because the assumption $\gamma<\frac{1}{2 k^{\max }}$ implies that

$$
1-\frac{1}{\hat{k}} \gamma \hat{k}^{2}-\frac{1}{\hat{k}} \gamma \rho \sigma^{2}>1-\frac{1}{2 \hat{k} k^{\max }} \hat{k}^{2}-\frac{1}{2 \hat{k} k^{\max }} \rho \sigma^{2}>\frac{1}{2}-\frac{1}{2} \frac{\sigma^{2}}{\hat{k} k^{\max }}>0,
$$

where the last inequality follows because $\hat{k} k^{\max } \geq \hat{k}\left(k^{\max }-\hat{k}\right)=\sum P(k) k k^{\max }-\hat{k}^{2} \geq$ $\sum P(k) k^{2}-\hat{k}^{2}=\sigma^{2}$. Furthermore, part b follows from part a.

To see part c, note that $4 \hat{k}-4 \gamma \hat{k}^{2}-\gamma^{2} \hat{k} \sigma^{2}-4 \gamma \rho \sigma^{2}+2 \gamma^{2} \hat{k} \rho \sigma^{2}-\gamma^{2} \hat{k} \rho^{2} \sigma^{2}$ is decreasing in $\rho$ and therefore,

$$
\begin{aligned}
4 \hat{k}-4 \gamma \hat{k}^{2}-\gamma^{2} \hat{k} \sigma^{2}-4 \gamma \rho \sigma^{2}+2 \gamma^{2} \hat{k} \rho \sigma^{2}-\gamma^{2} \hat{k} \rho^{2} \sigma^{2} & >4 \hat{k}-4 \gamma \hat{k}^{2}-\gamma^{2} \hat{k} \sigma^{2}-4 \gamma \sigma^{2}+2 \gamma^{2} \hat{k} \sigma^{2}-\gamma^{2} \hat{k} \sigma^{2} \\
& =4\left(\hat{k}-\gamma \hat{k}^{2}-\gamma \sigma^{2}\right)>0
\end{aligned}
$$

where the last inequality follows from part a. Finally, to prove claim 4 , it sufficient to show the following: (1.) $\eta(-1)>0$, (2.) $\eta(0)<0$, (3.) $\frac{d^{2} \eta}{d \rho^{2}}>0$ for $\rho \in[-1,1]$ and (4.) $\eta(\rho) \leq 0$ 
for $\rho \in(0,1)$. Point 1 and 2 are immediate from substituting $\rho=-1$ and $\rho=0$ in $\eta(\rho)$ respectively. To show point 3 note that

$$
\begin{aligned}
\frac{d^{2} \eta}{d \rho^{2}} & =-6 \hat{k}^{2} \sigma^{2} \gamma^{2}+12 \hat{k} \sigma^{2} \gamma-12 \sigma^{4} \gamma^{2} \rho \\
& >-6 \hat{k}^{2} \sigma^{2} \gamma^{2}+12 \hat{k} \sigma^{2} \gamma-12 \sigma^{4} \gamma^{2} \\
& =6 \gamma \sigma^{2}\left(2 \hat{k}-\hat{k}^{2} \gamma-2 \sigma^{2} \gamma\right)>0
\end{aligned}
$$

where the proof for the last inequality follows from part a. Finally, given that $\frac{d^{2} \eta}{d \rho^{2}}>0$ for $\rho \in[-1,1]$, to show point 4 is sufficient to show that $\eta(1) \leq 0$, or that $-4 \hat{k}\left(\hat{k}-\hat{k}^{2} \gamma-\sigma^{2} \gamma\right)<0$ which follows from part a. 